\title{
REVIEWS
}

Check for updates

\section{The biological and clinical significance of emerging SARS-CoV-2 variants}

Kaiming Tao ${ }^{1,7}$, Philip L. Tzou 1,7, Janin Nouhin (1) ', Ravindra K. Gupta ${ }^{2}$, Tulio de Oliveira ${ }^{3}$, Sergei L. Kosakovsky Pond ${ }^{4}$, Daniela Fera $\mathbb{1}^{5}$ and Robert W. Shafer (10 ${ }^{1,6 凶}$

Abstract | The past several months have witnessed the emergence of SARS-CoV-2 variants with novel spike protein mutations that are influencing the epidemiological and clinical aspects of the COVID-19 pandemic. These variants can increase rates of virus transmission and/or increase the risk of reinfection and reduce the protection afforded by neutralizing monoclonal antibodies and vaccination. These variants can therefore enable SARS-CoV-2 to continue its spread in the face of rising population immunity while maintaining or increasing its replication fitness. The identification of four rapidly expanding virus lineages since December 2020, designated variants of concern, has ushered in a new stage of the pandemic. The four variants of concern, the Alpha variant (originally identified in the UK), the Beta variant (originally identified in South Africa), the Gamma variant (originally identified in Brazil) and the Delta variant (originally identified in India), share several mutations with one another as well as with an increasing number of other recently identified SARS-CoV-2 variants. Collectively, these SARS-CoV-2 variants complicate the COVID-19 research agenda and necessitate additional avenues of laboratory, epidemiological and clinical research.

Humoral immunity Immunity mediated via host antibodies including those that directly neutralize virus as well as those that recruit other host immune functions.

Viral mutation rate The rate of mutation calculated in vitro as the number of nucleotide incorporation errors per round of replication or in vivo as the number of nucleotide changes observed during a fixed time such as 1 year. Although the two rates are related, the in vivo rate is also influenced by the number of replication cycles that occur over time and the frequency with which multiple mutations occur at the same position. 凶e-mail: rshafer@stanford.edu https://doi.org/10.1038/ s41576-021-00408-x
Among the many unprecedented aspects of the SARS-CoV-2 pandemic is the intense virological monitoring that has occurred, with more than two million virus isolates having undergone partial or complete genomic sequencing. Initially, genetic sequencing suggested that SARS-CoV-2 was exceptionally well adapted to humans, spreading rapidly with little evidence for natural selection among circulating viruses. This changed during the later months of 2020, with the first reports of emergent SARS-CoV-2 variants associated with increased transmissibility, disease severity and escape from humoral immunity.

In this Review, we create a framework for understanding SARS-COV-2 variants by describing fundamental aspects of SARS-CoV-2 evolution, the structure and function of the SARS-CoV-2 spike protein and the laboratory methods used to characterize spike variants. We then describe the biological properties and epidemiological characteristics of these variants and their associated mutations. Lastly, we describe the types of study required for the research, clinical and public health communities to respond to the new threat posed by emerging SARS-CoV-2 variants. Given the wide public interest in this topic, we provide a box of key points. We also provide a repository of the SARS-CoV-2 variant neutralization data discussed in this Review (Stanford University Coronavirus Antiviral \& Resistance Database - Susceptibility Data).

\section{SARS-CoV-2 evolution}

Coronaviruses contain an exonuclease enzyme that reduces their replication error rate by about 15 -fold to 20 -fold in vitro, resulting in an in vivo viral mutation rate about 10 -fold lower than that of influenza ${ }^{1-3}$. Nonetheless, they accumulate mutations and generate further diversity through the process of recombination when variants with different mutations infect the same host ${ }^{4-6}$. Recombination between different SARS-related coronaviruses is likely to have led to the emergence of SARS-CoV-2 (REF.') and, although it can be difficult to detect owing to the similarity of most sequences, recombination is occurring to some extent among circulating SARS-CoV-2 variants ${ }^{6,8}$. Additionally, host-mediated RNA editing by APOBEC and ADAR enzymes, as evidenced by the dominance of $\mathrm{C}$ to $\mathrm{U}$ changes in specific dinucleotide contexts, contributes to SARS-CoV-2 diversity $^{9,10}$.

Although it had been previously assumed that waning immunity explained the observation that people are commonly reinfected with endemic common-cold coronaviruses $^{11}$, recent studies suggest that antigenic drift also contributes to the lack of long-lasting protection following coronavirus infections ${ }^{12,13}$. HCoV-229E and $\mathrm{HCoV}$-OC43 sequences over a 30 -year period demonstrate a ladder-like phylogenetic tree topology consistent with the emergence of novel variants sweeping 


\section{Key points \\ - The past several months have witnessed the emergence of four SARS-CoV-2 variants of concern (Alpha, Beta, Gamma and Delta) associated with increased transmissibility, increased risk of reinfection and/or reduced vaccine efficacy. \\ - Many additional SARS-CoV-2 variants sharing mutations and biological features with these variants are also increasingly being identified. \\ - The increasing number of SARS-CoV-2 variants share a repertoire of mutations that is enabling the virus to spread despite rising population immunity while maintaining or increasing its replication fitness. \\ -Whereas most emerging mutations reduce the protective effects of neutralizing antibodies generated by infection and vaccination, several recently identified mutations appear to antagonize the innate immune response to initial infection. \\ - The emergence of SARS-CoV-2 variants requires an expanded research agenda to improve our understanding of emerging SARS-CoV-2 mutations and the correlates of protective immunity against variants with these mutations.}

APOBEC and ADAR

enzymes

Apolipoprotein B mRNA-

editing enzyme catalytic

polypeptide-like (APOBEC)

and adenosine deaminase acting on RNA (ADAR) are

host enzymes that edit viral genomes. Although these enzymes represent an antiviral defence mechanism, it is often hypothesized that these enzymes can contribute to viral evolution through the human population similar to seasonal influenza, albeit at a slower rate, with virus isolates from one time point often evading neutralization by plasma from persons infected several years earlier ${ }^{12,13}$.

The evolutionary rate of SARS-CoV-2 has been estimated to be between 0.0004 and 0.002 mutations per nucleotide per year ${ }^{14-19}$. Although the possibility that synonymous mutations may influence SARS-CoV-2 phenotypic properties should not be discounted, there have been no reports of this phenomenon occurring within the SARS-CoV-2 spike gene. Therefore, in this Review, we use the term mutation to indicate an amino acid change from the Wuhan-Hu-1 reference sequence (GenBank accession: NC_045512.2).

The phylogenetic classification of emergent SARSCoV-2 lineages has been difficult because new lineages often differ from one another by just a few nucleotides ${ }^{20,21}$. Geographical classification has been challenging because most variants have been detected in multiple countries and there are marked disparities in the proportion of viruses undergoing sequencing in different countries. Two commonly used systems have been developed for epidemiological surveillance: the Phylogenetic Assignment of Named Global Outbreak (PANGO) lineage ${ }^{22}$ and NextStrain ${ }^{23}$ systems. The PANGO lineage system provides greater specificity and is used more frequently. It contains an alphabetical prefix and a suffix containing up to three numbers separated by periods indicating sub-lineages (such as B.1.1.7). However, as the system allows for only three hierarchical levels, the introduction of a new lineage suffix can make it difficult to identify the ancestral lineage of a variant.
In addition, the lineage of a virus does not always correspond to its component mutations, as a virus can acquire additional biologically relevant mutations without being assigned to a new lineage.

The first indication of SARS-CoV-2 genetic evolutionary selection pressure became evident as a novel virus variant containing the spike mutation D614G emerged in early 2020 and rose to a prevalence of nearly $100 \%$ by June 2020 (REFS ${ }^{8,24-26}$ ). By the end of 2020 and in early 2021, several variants with recurrent mutations (in addition to $\mathrm{D} 614 \mathrm{G}$ ) occurring primarily, but not exclusively, in the spike protein were also reported. In December 2020, B.1.1.7, a rapidly growing lineage in the UK associated with an unexpectedly large number of genetic changes, was reported on the virological.org discussion forum ${ }^{27}$. Retrospective analyses determined that the earliest clinical sample of this variant had been obtained in the UK in late September 2020.

Within 1 month, two additional rapidly growing lineages with large numbers of genetic changes were reported from South Africa ${ }^{16}$ and Brazil ${ }^{19}$. The B.1.351 variant rose in prevalence in South Africa from $11 \%$ in October to $87 \%$ by December ${ }^{28}$. The P.1 variant emerged in Manaus, Brazil, a region that was estimated to have achieved an infection rate approaching $75 \%$ by October 2020 , but which experienced a surge in new cases beginning in November 2020 (REFS ${ }^{19,29,30}$ ). Subsequently, a novel variant (B.1.617.2) increased in prevalence from $2 \%$ in February 2021 to $87 \%$ in May 2021 in Maharashtra, India, as India experienced a dramatic surge in cases ${ }^{31}$. Since then, the B.1.617.2 variant has spread widely in multiple countries ${ }^{32-34}$ and displayed evidence of being even more transmissible than the B.1.1.7 variant, and is likely to cause more severe disease than earlier virus variants $^{35,36}$.

Variants that have spread widely and displayed evidence for being more transmissible, causing more severe disease and/or reducing neutralization by antibodies generated during previous infection or vaccination have been classified as variants of concern (VOCs) by the World Health Organization (WHO) ${ }^{37}$, US Centers for Disease Control and Prevention (CDC) ${ }^{38}$ and COVID-19 Genomics UK Consortium (COG-UK) ${ }^{39}$. Variants that have spread less widely but contain mutations similar to those present within VOCs have been classified as variants of interest (VOIs). On 31 May 2021, the WHO labelled VOCs and VOIs using the Greek alphabet, with the current VOCs designated as Alpha (B.1.1.7), Beta (B.1.351), Gamma (P.1) and Delta (B.1.617.2) (FIG. 1).

\section{Spike structure and immune epitopes}

The SARS-CoV-2 spike protein is a 1,273-amino acid trimeric glycoprotein responsible for virus entry into host cells (FIG. 1). Each spike monomer has a largely exposed S1 attachment domain (residues 1-686) and a partially buried S2 fusion domain (residues 687-1,273) (0,41 $^{\text {. Part }}$ of $\mathrm{S} 1$, called the receptor-binding domain (RBD; residues 306-534), alternates between a closed/down position and an open/up position. When in the up position, it binds to the human angiotensin-converting enzyme 2 (ACE2) receptor, the necessary first step for entry into most, if not all, cells ${ }^{42-45}$. Approximately 20 RBD residues form 

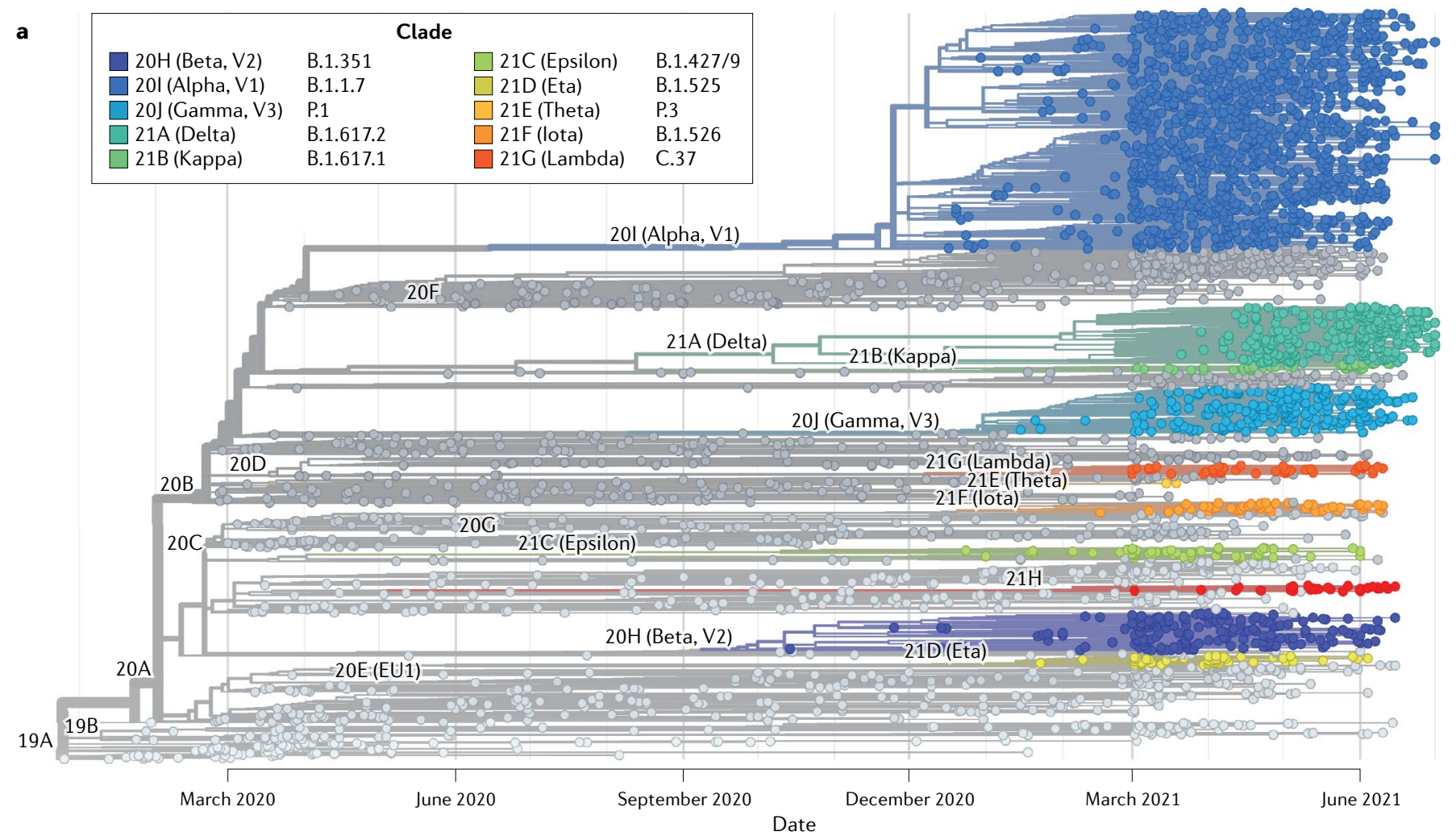

b

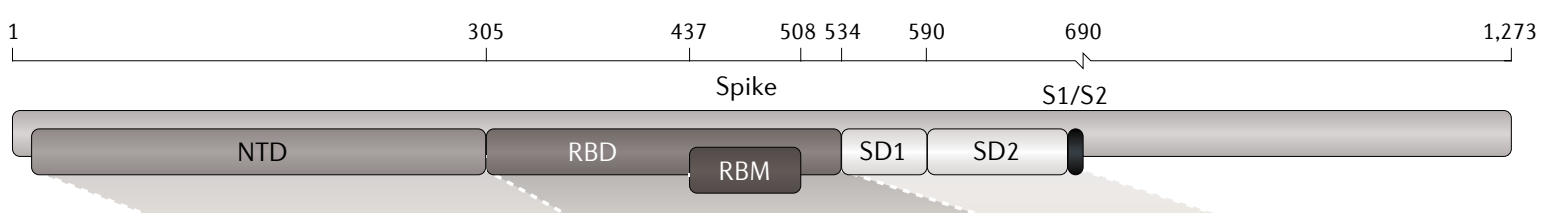

\begin{tabular}{|c|c|c|c|c|c|c|c|c|c|c|c|c|c|c|c|c|c|c|}
\hline WHO & $\begin{array}{l}\text { PANGO } \\
\text { lineage }\end{array}$ & $\begin{array}{l}\text { Country } \\
\text { of first } \\
\text { identification }\end{array}$ & 18 & 69 & 95 & 144 & 242 & 417 & 452 & 478 & 484 & 490 & 501 & 614 & 681 & 701 & $\begin{array}{l}\text { nsp6: } \\
106\end{array}$ & $\begin{array}{l}N: 203 / \\
204\end{array}$ \\
\hline$\square$ Alpha & B.1.1.7 & UK & & del & & del & & & & & & & Y & G & $\mathrm{H}$ & & del & $K / R$ \\
\hline$\square$ Gamma & P.1 & Brazil & $\mathrm{F}$ & & & & & $\mathrm{T}$ & & & K & & Y & G & & & del & $\mathrm{K} / \mathrm{R}$ \\
\hline$\square$ Delta & B.1.617.2 & India & & & 1 & & & & $\mathrm{R}$ & K & & & & G & $\mathrm{R}$ & & & $M$ \\
\hline$\square$ Theta & P.3 & Philippines & & & & & & & & & K & & Y & G & $\mathrm{H}$ & & & $\mathrm{K} / \mathrm{R}$ \\
\hline$\square$ lota & B.1.526 & USA & & & 1 & del & & & & & K & & & G & & V & del & \\
\hline$\square$ Карра & B.1.617.1 & India & & & 1 & & & & $R$ & & Q & & & G & $\mathrm{R}$ & & & M \\
\hline$\square$ Zeta & P.2 & Brazil & & & & & & & & & K & & & $G$ & & & & $K / R$ \\
\hline$\square$ Lambda & С. 37 & Peru & & & & & & & $\mathrm{Q}$ & & & S & & G & & & del & $K / R$ \\
\hline
\end{tabular}

Fig. 1 | SARS-CoV-2 variants: evolution and constituent mutations. a | Phylogenetic tree based on subsampling of globally circulating sequences created by NextStrain (CC BY 4.0). The tree shows that nearly all variants of concern (VOCs; Alpha, Beta, Gamma and Delta) and variants of interest (VOIs; Kappa, Epsilon, Eta, Theta, lota and Lambda) emerged independently beginning in late 2020. b | The most common mutations present in multiple VOCs and VOIs. Numeric column headers indicate spike protein positions except for two non-spike mutations in the nsp6 and nucleocapsid (N) genes. The second row indicates the residue found in the reference sequence.
Spike protein residues are mapped to their associated domain within the spike protein, as shown in various shades of grey above the table. Deletions are indicated 'del'. Several additional mutations in other viral proteins also appear to have arisen more than once, including orf3a:Q57H and nsp2:T85I. NTD, amino-terminal domain; PANGO, Phylogenetic Assignment of Named Global Outbreak; RBD, receptor-binding domain; RBM, receptor-binding motif; SD, subdomain; S1/S2, junction between the exposed S1 attachment domain and the partially buried S2 fusion domain; WHO, World Health Organization. 


\section{Neutralizing antibodies Antibodies that alone can prevent virus infection of cells in vitro. Neutralization is determined almost entirely by an antibody fragment antigen-binding (Fab) region.}

Epitope

An antigenic determinant of a protein. B lymphocyte antibody epitopes are often formed by amino acids from different parts of a protein that are brought together during protein folding. T lymphocyte epitopes are linear peptides recognized by T lymphocyte receptors when bound to a human leukocyte antigen (HLA) protein on a cell surface. As human HLA proteins are heterogenous, different people recognize different epitopes of the same protein. contacts with the human ACE2 receptor. The part of the $\mathrm{RBD}$ containing these residues encompasses residues 438-506 and is called the receptor-binding motif (RBM), whereas the remainder of the RBD is called the RBD core.

Similar to the RBD, much of the S1 amino-terminal domain (NTD) is also exposed on the $S$ trimer surface. The remainder of S1 contains two subdomains downstream of the RBD traditionally referred to as subdomain 1 (SD1) and subdomain 2 (SD2) that we refer to in this Review as the $\mathrm{S} 1$ carboxy-terminal domain (CTD). The spike protein also has 22 glycosylation sites, distributed among both the S1 and S2 domains. Within S1, eight of the glycans are found in the NTD, two are in the RBD core, three are in the CTD and nine are in S2 (REF. ${ }^{46}$.

S1 displays more amino acid variability than S2 among SARS-related coronaviruses (FIG. 2). Within S1, the RBD and the NTD are more variable than the CTD. Within the RBD, the RBM is more variable than the RBD core. As of June 2021, 42 spike mutations have a global sampled prevalence $\geq 1.0 \%$ including 15 in the NTD, 6 in the RBD, 5 in the CTD and 9 in S2 (Supplementary Table 1). Most of the $32 \mathrm{~S} 1$ mutations with a prevalence $\geq 1.0 \%$ arose in multiple SARS-CoV-2 lineages.

The spike RBD is the main target of neutralizing antibodies $^{47-52}$. The presence of neutralizing monoclonal antibodies (mAbs) targeting the RBD correlates with protection in animal models and in previously infected and vaccinated persons, although cellular immune responses and potentially non-neutralizing antibodies are likely to have contributed to protection in these studies $^{53-59}$. The development of neutralizing antibodies early in the course of infection has been associated with lower virus levels and greater protection from severe infection $^{58,60-63}$. Finally, the passive administration of neutralizing $\mathrm{mAbs}$ reduces the severity of infection when administered early ${ }^{62,64-66}$.
High-resolution X-ray crystallography and cryo-electron microscopy structures have been published for more than $100 \mathrm{mAbs}$, including 5 with US Food and Drug Administration (FDA) Emergency Use Authorizations (EUAs) and several additional mAbs in phase III clinical trials ${ }^{67}$. Most mAbs target either the RBD RBM or the RBD core; several target the NTD. Those targeting the RBM compete with RBD binding to ACE2. Those targeting the RBD core often cross-neutralize other SARS-related coronaviruses ${ }^{49,68,69}$. The NTD-targeting neutralizing antibodies primarily bind a single epitope comprising the largest glycan-free surface facing away from the viral membrane referred to as the NTD supersite ${ }^{51,52,70}$.

Several classification schemes have been developed to describe RBD-binding mAbs based upon whether they bind the RBM or RBD core, whether they bind the RBD in its up and/or down configuration and the extent to which they compete with other $\mathrm{mAbs}^{71-74}$. Among those mAbs targeting the RBM, one group binds epitopes that overlap extensively with the ACE2 binding site and, as a result, binds solely when the RBD is in the open state. This group, which is referred to as class $1 \mathrm{mAbs}$, is typically encoded by the closely related IGHV3-53 and IGHV3-66 heavy chain genes and has short complementarity-determining region $\mathrm{H} 3$ loops. The second main group of RBM-binding $\mathrm{mAbs}$ (class 2) has a smaller ACE2 binding footprint and, as a result, can often bind the RBD in the closed state. Several other RBM-binding $\mathrm{mAbs}$ are more difficult to classify, including a class that binds a quaternary epitope involving more than one $\mathrm{RBD}^{71,72}$.

Antibodies that target the RBD core also form two major clusters, one on the surface-accessible face of the RBD and another whose epitope is buried in the closed state ${ }^{71-74}$. Antibodies that bind to the surface-accessible face of the RBD core can bind in either

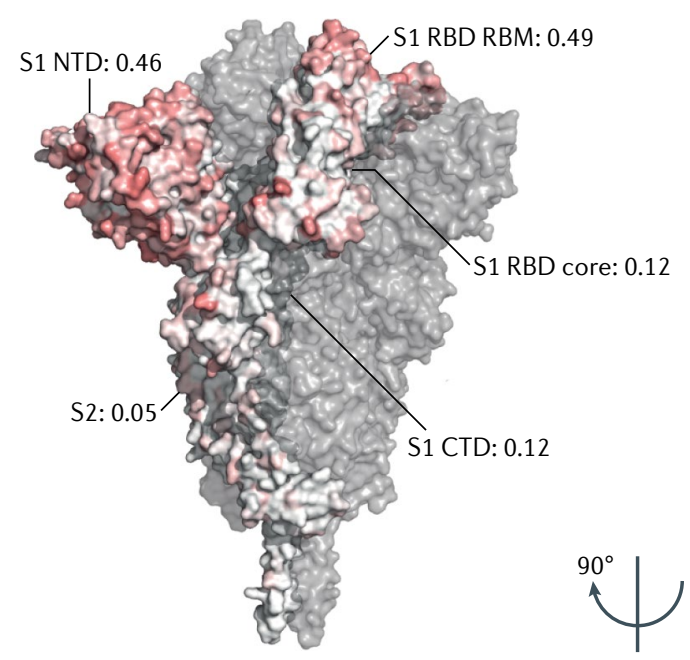

Fig. 2 | Genetic variability of the SARS spike proteins. Position-specific sequence variability and median domain-specific pairwise distances among SARS-related coronaviruses. Results were derived from an alignment of 24 representative sarbecovirus spike sequences having a nucleotide genetic distance (TN93 model) of $\geq 0.01$. Position-specific entropy is superimposed for one of three monomers on a surface representation of trimeric SARS-CoV-2 spike (Protein Databank (PDB) code: 6XR8), with white indicating conserved residues and the shade of red

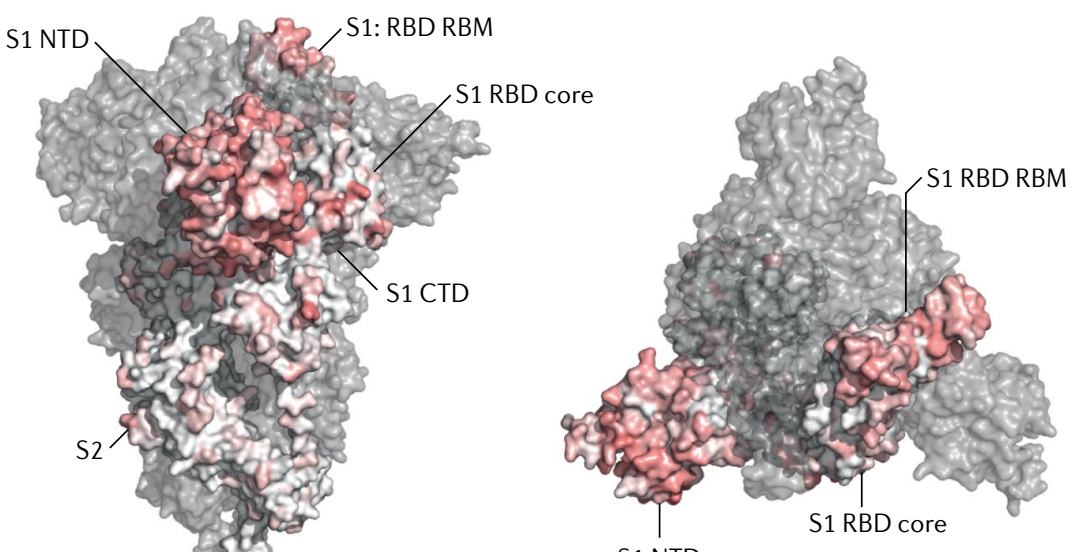

S1 NTD

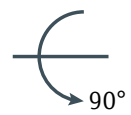

indicating the extent of sequence variability. Two $90^{\circ}$ rotated side views (left and middle panels) and one top view (right panel) of the spike trimer are shown. The median pairwise distance among SARS-related coronaviruses is greatest for the $\mathrm{S} 1$ amino-terminal domain (NTD) and receptor-binding domain (RBD). Within the $\mathrm{RBD}$, the median pairwise distance is greater for the receptor-binding motif (RBM) than for the core region. CTD, carboxy-terminal domain; S1, exposed attachment domain; S2, partially buried fusion domain. 
a RBM class 1

$\begin{array}{ll}\text { - Casirivimab } & \cdot \text { BD-629 } \\ \text { - Etesevimab } & \cdot \text { C103 } \\ \text { - Tixagevimab } & \cdot \text { C105 } \\ \text { - Regdanvimab } & \cdot \text { CC12.1 } \\ \text { - S2E12 } & \cdot \text { COVA2-04 } \\ \text { - CC12.3 } & \text { - COVA2-39 } \\ \text { - B38 } & \text { - CV07-250 } \\ \text { - BD-236 } & \text { - CV30 } \\ \text { - BD-604 } & \cdot \text { S2H14 }\end{array}$

b RBM class 2
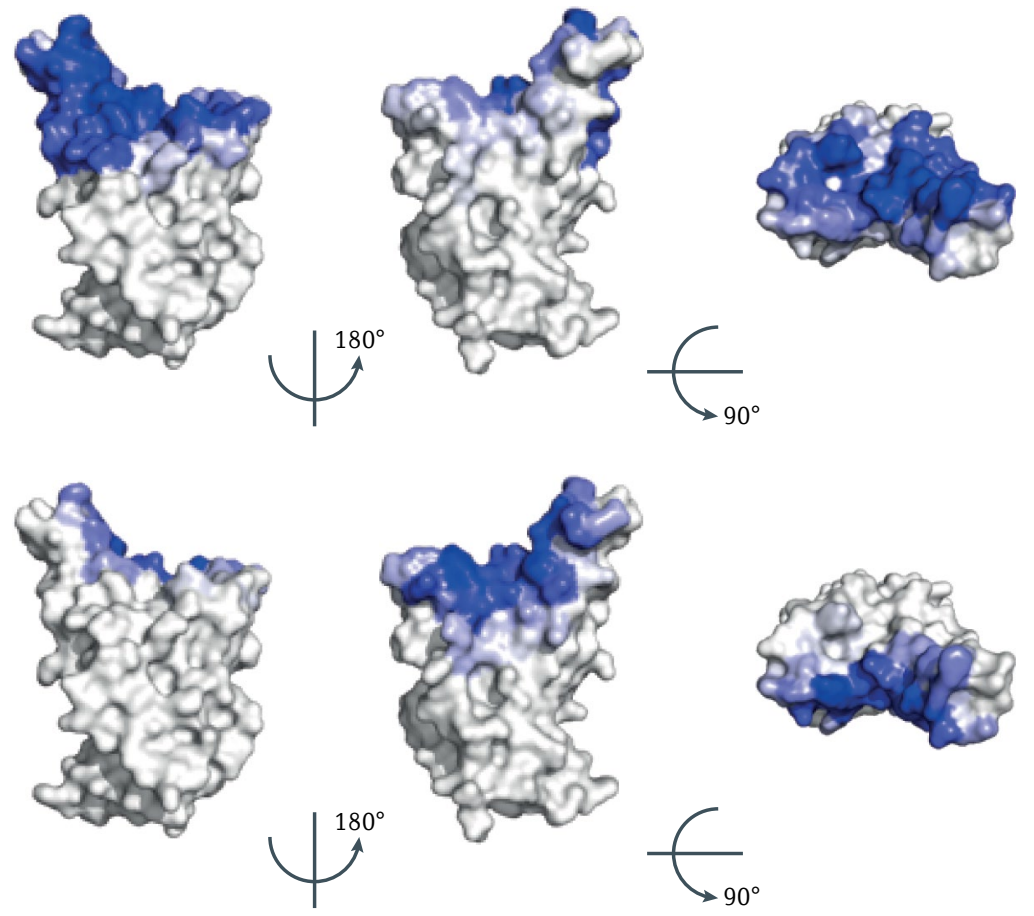$$
\text { 西 }
$$

C RBD core 1

$$
\begin{aligned}
& \text { - C104 } \\
& \text { - CV07-270 } \\
& \text { - H11-D4 } \\
& \text { - H11-H4 } \\
& \text { - S2H13 } \\
& \text { - Sb23 } \\
& \text { - Ty1 }
\end{aligned}
$$
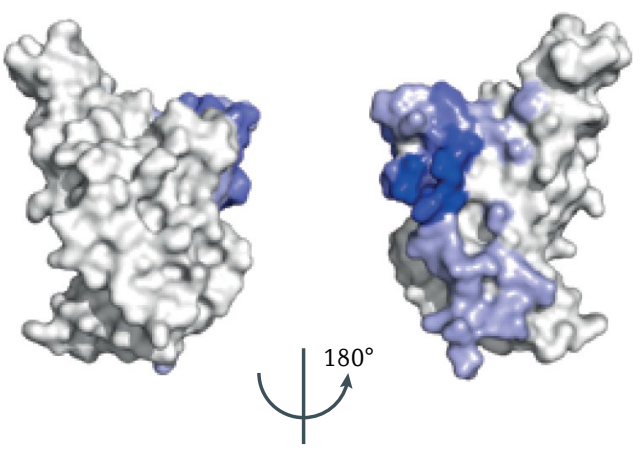

\section{d RBD core 2}

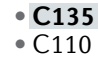

$\begin{array}{ll}\text { - Imdevimab } & \bullet \text { C135 } \\ \text { - Sotrovimab } & \bullet \text { C110 }\end{array}$

$\begin{array}{ll} & \\ & \\ & \\ \text { - CR3022 } & \text { S S2A4 } \\ \text { - EY6A } & -S 304 \\ \text { - H014 } & \end{array}$
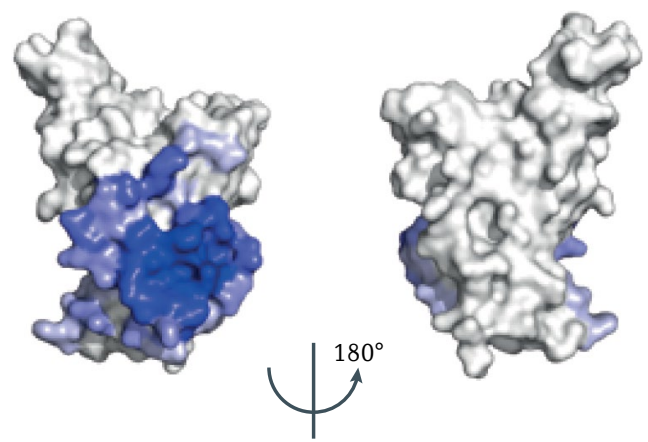

Fig. 3 | SARS-CoV-2 spike-targeted antibody classifications. Classification of monoclonal antibodies (mAbs) targeting the SARS-CoV-2 spike receptor-binding domain (RBD) epitopes. For the two classes of mAbs binding the receptor-binding motif (RBM), $90^{\circ}$ rotated side views and one top view of a surface RBD representation are shown (parts a,b). For the two classes of RBD core-binding mAbs, just the $90^{\circ}$ rotated side views are shown (parts c,d). Each image derived from coordinates of the Protein Databank (PDB) structure 6M0J. Bold highlighted mAbs are in phase Ill clinical trials (as of July 2021). Blue intensity is proportional to the number of $m A b s$ binding to the underlying amino acid residues. RBM refers to the region of the RBD containing the angiotensin-converting enzyme 2 (ACE2)-binding residues. RBM class $1 \mathrm{mAbs}$ (part a) bind the RBD only in its up position, whereas RBM class $2 \mathrm{mAbs}$ (part b) can bind the RBD in its up or down position. A third RBM mAb class binds to a quaternary epitope comprising more than one RBD but is not shown as it would require the trimeric spike. Epitopes for amino-terminal domain-binding mAbs are not shown.

the open or closed state and those that target the RBD core epitope bind only in the open state. FIGURE 3 displays the epitopes of those mAbs with high-resolution structures that are either in advanced clinical trials or have been assessed for their activity against viruses with mutant spike proteins. Supplementary Table 2 
Fc-effector functions

Antibody functions mediated

by their fragment crystallizable

(Fc) region, including

complement activation,

antibody-dependent

cellular cytotoxicity and

antibody-dependent cellular

phagocytosis. These functions

may be particularly important

for eliminating virally infected

cells. They are more difficult

to study in vitro than in vivo.

Convalescent plasma

Plasma samples from persons previously infected with

SARS-CoV-2 usually contain neutralizing antibodies that

bind to the SARS-CoV-2 spike protein and prevent it from

infecting cells in vitro.

Convalescent plasma samples

are characterized by the

number of months since

recovery from infection, the

severity of illness associated

with infection, and the extent

to which the plasma can be

diluted and still retain the

ability to prevent infection

in vitro against different

SARS-CoV-2 variants.

Deep mutational scanning A method that makes use of next-generation sequencing to measure in a single experiment the activity of many unique variants of a protein describes the epitopes of those mAbs being studied in clinical trials.

In addition to RBD-targeting and NTD-targeting antibodies, there may be a protective role for neutralizing antibodies targeting other parts of the spike and for non-neutralizing antibodies. Several neutralizing antibodies bind the S1 CTD and S2 of multiple coronaviruses, although these have been much less potent than RBD-targeting and NTD-targeting $\mathrm{mAbs}^{75-77}$. Non-neutralizing antibody Fc-effector functions such as complement activation, cellular cytotoxicity and phagocytosis have also been shown to afford protection in animal models ${ }^{78,79}$ and in the initial weeks following vaccine administration ${ }^{80}$. However, the protection afforded by non-neutralizing antibodies in the absence of neutralizing antibodies is difficult to quantify $^{81,82}$

The early development of cytotoxic T lymphocyte (CTL) responsiveness in persons infected with SARS-CoV-2 correlates with less severe COVID-19 illness ${ }^{83,84}$. CTL responses also contribute to protection from severe infection in non-human primates in the presence of low titres of neutralizing antibodies ${ }^{53,85}$ and in persons with impaired humoral immunity ${ }^{86}$. SARS-CoV-2 infection and immunization with the spike protein elicits helper T lymphocyte and CTL responses ${ }^{58,87,88}$. Indeed, many studies have identified specific human leukocyte antigen (HLA)-restricted helper T cell and CTL spike epitopes ${ }^{58,89,90}$. Analyses of peptide libraries from several VOCs have shown that, with a few exceptions, spike CTL epitopes either remain unchanged or able to bind most HLA molecules ${ }^{91,92}$. Nonetheless, the number of $\mathrm{T}$ cell spike epitopes recognized by a person varies according to their HLA profile and is much lower than the total number of SARS-CoV-2 $\mathrm{T}$ cell epitopes ${ }^{93,94}$. Mutations at $\mathrm{T}$ cell epitopes have also been observed within several cohorts with the common HLA type $\mathrm{A}^{\star} 02$ (REF. ${ }^{95}$ ) and at low levels within the circulating viruses of individual patients ${ }^{96}$.

In vitro selection and neutralization experiments An increasing number of studies have described either the in vitro selection of SARS-CoV-2 immune escape spike mutations or the impact of mutations on the neutralizing activity of mAbs, convalescent plasma or plasma from vaccinated persons $s^{52,97,98}$. Studies of plasma from patients who were previously infected provide insight into the risk of reinfection with a SARS-CoV-2 variant, whereas those from immunized persons are relevant to vaccine efficacy. Although most convalescent plasma samples studied so far were obtained prior to the emergence of immune escape variants ('pre-variant isolates'), more recent studies have studied plasma from patients infected with different VOCs ${ }^{99-104}$.

In vitro selection experiments have been performed most commonly using non-replicative pseudotyped or replication-competent chimeric viruses ${ }^{105-108}$. SARS-CoV-2 pseudo-typed viruses are produced by co-transfecting a SARS-CoV-2 protein expression vector and a construct encoding the components required for replication of a different virus that lacks the coding sequence for their own surface protein - most commonly vesicular stomatitis virus (VSV), HIV-1 or murine leukaemia virus. These constructs also encode a reporter gene such as luciferase or green fluorescent protein. Chimeric viruses contain the SARS-CoV-2 spike sequence in a VSV genome lacking the sequence for the VSV surface protein ${ }^{107,108}$. VSV-based chimeric viruses are particularly useful for mutation selection studies because they can undergo multiple rounds of replication. There have also been reports describing the in vivo selection of spike mutations in animal models ${ }^{109}$ and in persons infected with SARS-CoV-2 with prolonged infections or receiving $\mathrm{mAbs}^{109-114}$.

Neutralization studies compare the ability of a mAb or plasma sample to inhibit the cellular entry of a virus containing one or more spike mutations with viruses lacking these mutations. Neutralization studies have been performed using either pseudo-typed viruses, VSV-based chimeric viruses, full-length cloned recombinant SARS-CoV-2 viruses ${ }^{115-120}$ or low-passage or plaque-purified cultured isolates ${ }^{121-124}$. Pseudo-typed and chimeric viruses have been used most frequently because it is simpler to introduce mutations into a plasmid encoding just the spike gene compared with using a clinical virus isolate or a recombinant virus generated using a system that requires either multiple plasmids or bacterial or yeast artificial chromosomes ${ }^{119,120}$. Although the results of neutralization experiments using pseudo-typed viruses, VSV-based chimeric viruses and full-length SARS-CoV-2 usually yield similar results ${ }^{97,107,125,126}$, full-length viruses are expected to be more reliable and can be studied in animal models ${ }^{127}$.

The effects of nearly all individual RBD mutations on protein expression in yeast (a correlate of protein folding stability), ACE2 binding and binding to a wide variety of $\mathrm{mAbs}$ and plasma samples have also been assessed using deep mutational scanning in which each yeast cell producing a different RBD mutation is labelled with a distinct genomic sequence ${ }^{50,128-131}$. Although this approach does not quantify the effect of RBD mutations on $\mathrm{mAb}$ neutralization, it has proved useful as a screening assay to identify mutations that require further study in cell culture. High-throughput biochemical assays such as enzyme-linked immunosorbent assays (ELISAs) are also being developed to allow clinical laboratories to measure the ability of plasma to inhibit ACE2 binding to RBDs belonging to different SARS-CoV-2 variants ${ }^{132-134}$.

In vitro neutralization experiments of SARS-CoV-2 variants are usually reported as a fold reduction in susceptibility compared with a control virus, such as the reference Wuhan-Hu-1 virus, variants containing just the D614G mutation or other pre-variant isolates ${ }^{97}$. In the sections that follow, we summarize the neutralization susceptibility of different viruses as being $<3$-fold, 3-10-fold and $>10$-fold reductions compared with the control virus as these thresholds represent approximately one-half-log and one-log reductions in susceptibility. Nonetheless, the absolute level of neutralization is often more clinically relevant than the fold change compared with a control virus as the same reduction in susceptibility to a mAb or to vaccinee plasma will be more consequential for $\mathrm{mAbs}$ with low intrinsic activity or vaccines that do not elicit high levels of neutralizing 
antibodies. In summarizing the results of neutralizing experiments, we have also pooled results obtained using pseudo-typed, chimeric and infectious viruses as the results of these assays are usually concordant ${ }^{97,107,125,126}$.

Of the more than 6,000 plasma samples from persons receiving a complete course of vaccination, $76 \%$ were obtained from persons receiving an authorized mRNA vaccine (Pfizer/BioNTech BNT162b (54\%) and Moderna mRNA-1273 (22\%)), 9\% from recipients of the adenovirus-vectored AstraZeneca AZD1222 vaccine, $6 \%$ from recipients of the inactivated Sinovac CoronaVac vaccine, $3 \%$ from recipients of the adenovirus-vectored Janssen Ad26.CoV2.s vaccine, 3\% from recipients of the inactivated Bharat Biotech Covaxin (BBV152) vaccine and $1 \%$ each from recipients of the protein subunit Novavax NVX-CoV2373, the adenovirusvectored Gamaleya Research Institute Sputnik V and the inactivated Sinopharm BBIBP-CorV vaccines.

\section{SARS-CoV-2 mutations}

Currently circulating SARS-CoV-2 VOCs and VOIs share several mutations that enable them to spread in the face of rising population immunity while maintaining or increasing their replication fitness. These mutations belong to a repertoire of recurrent mutations, most of which are in the spike gene. FIGURE 4 illustrates the most biologically and clinically significant spike mutations and their change in prevalence since the early stages of the pandemic. To understand the biological properties and epidemiological characteristics of the increasing number of SARS-CoV-2 VOCs and VOIs, it is necessary to understand their component mutations. Here, we divide these mutations into seven categories: D614G; the RBD mutation N501Y; the RBD mutation E484K; other RBD mutations; NTD mutations; mutations proximal to the S1/S2 furin cleavage site; and non-spike mutations.

\section{D614G}

The prevalence of the D614G mutation began increasing in late February 2020, and within several months it outcompeted all ancestral viruses and rose to a global prevalence approximating $100 \%{ }^{8}$. Infectious virus clones with D614G replicated to higher levels in primary human airway cells and in the upper respiratory tracts of hamsters ${ }^{135-137}$. D614G-containing virus clones were also associated with increased transmission between hamsters ${ }^{136}$. Cryo-electron microscopy studies have shown that D614G disrupts one or more interprotomer contacts, resulting in a greater likelihood that one or more of the three RBDs are in an open versus closed position and, hence, compatible with ACE2 receptor binding ${ }^{138,139}$. Subsequently, additional mutations in the spike CTD and in S2 have also been reported to possibly increase SARS-CoV-2 replication by a similar mechanism ${ }^{140}$. D614G may also be responsible for increasing the number of spike proteins per virion ${ }^{141,142}$ and the rate of S1/S2 cleavage ${ }^{143}$. Viruses with D614G have been slightly more susceptible to neutralization by mAbs, convalescent plasma and plasma from vaccinated individuals in some studies ${ }^{138,144}$ and slightly more resistant to neutralization in other studies ${ }^{136,145}$.

\section{N501Y}

$\mathrm{N} 501 \mathrm{Y}$ is present in the Alpha, Beta and Gamma VOCs. N501Y increases ACE2 affinity ${ }^{128,146,147}$ and increases virus replication in human upper-airway cells and in the upper respiratory tracts of hamsters ${ }^{127}$. N501Y does not influence the binding and neutralization of most $\mathrm{mAbs}^{48,109,117,121,148,149}$ (TABLE 1). Alone, it is also rarely associated with reduced susceptibility to convalescent plasma ${ }^{121,148-150}$ or plasma from persons receiving one of the two authorized mRNA vaccines (Pfizer/BioNTech BNT162b2 or Moderna mRNA-1273) or the Novavax NVX-CoV2373 protein subunit vaccine $\mathrm{e}^{115,121,148,150-153}$ (FIG. 5).

\section{E484K}

E484 is recognized by a high proportion of the polyclonal antibodies developing within persons infected with SARS-CoV-2 $\left(\mathrm{REF}^{5}{ }^{5}\right)$. E484K is present in the Beta and Gamma VOCs ${ }^{16,19}$ and in the VOIs Eta (B.1.525), Iota (B.1.526) ${ }^{154}$, Theta (P.3) $)^{155,156}$ and Zeta (p.2) ${ }^{157}$. E484K has also been reported within several Alpha variant sub-lineages ${ }^{158,159}$. E484Q has been reported in the Kappa VOI (B.1.617.1).

E484K has been selected in vitro by casirivimab and bamlanivimab and several other RBM class 1 and 2 $\mathrm{mAbs}^{48,105,110,130,160}$ and it reduces susceptibility to these $\mathrm{mAbs}^{64,105,109,117,121}$ (TABLE 1). E484K has resulted in 3-fold to 10 -fold reduced susceptibility to about $30 \%$ and $>10$-fold reduced susceptibility to about $10 \%$ of convalescent plasma samples ${ }^{121,130,160-162}$ (FIG. 5). E484K has also resulted in 3-fold to 10 -fold reduced susceptibility to about $30 \%$ of plasma samples from persons immunized with one of the authorized mRNA vaccines ${ }^{121,160-162}$ (FIG. 5).

\section{Other RBD mutations}

L452R is present in the Delta VOC, as well as the Kappa (B.1.617.1) and Epsilon (B.1.427/9) VOIs ${ }^{34,123}$. It reduces susceptibility to several RBM class $2 \mathrm{mAbs}$, including bamlanivimab, but not to the other FDA EUA-approved $\mathrm{mAbs}^{109,110,160,163}$ (TABLE 1). L452R has resulted in 3-fold to 10 -fold reduced susceptibility to about one third of convalescent and vaccinee plasma samples ${ }^{164-166}$ (FIG. 5). Pseudo-typed viruses containing L452R were associated with higher levels of cell entry in lung organoids compared with pseudo-typed viruses containing D614G alone but lower levels compared with pseudo-typed viruses containing N501Y (REF. ${ }^{123}$ ).

$\mathrm{K} 417 \mathrm{~N} / \mathrm{T}$ are present in the Beta (as $\mathrm{K} 417 \mathrm{~N}$ ) and Gamma (as K417T) VOCs. K417N/T rarely occur in the absence of other RBM mutations, possibly because K417 mutations appear to reduce ACE2 binding ${ }^{130,159}$. K417N confers $>100$-fold reduced susceptibility to etesevimab ${ }^{129}$ and about 10 -fold reduced susceptibility to casirivimab ${ }^{121}$ but retains susceptibility to bamlanivimab, imdevimab and sotrovimab ${ }^{121}$. K417N/T retain full susceptibility to plasma samples from persons previously infected with SARS-CoV-2 or immunized with one of the authorized mRNA vaccines ${ }^{117,121,167}$.

N439K increases ACE2 affinity ${ }^{128,168,169}$ and reduces imdevimab susceptibility ${ }^{129}$ (TABLE 1). Viruses containing $\mathrm{N} 439 \mathrm{~K}$ usually retain full susceptibility to convalescent 


\section{REVIEWS}

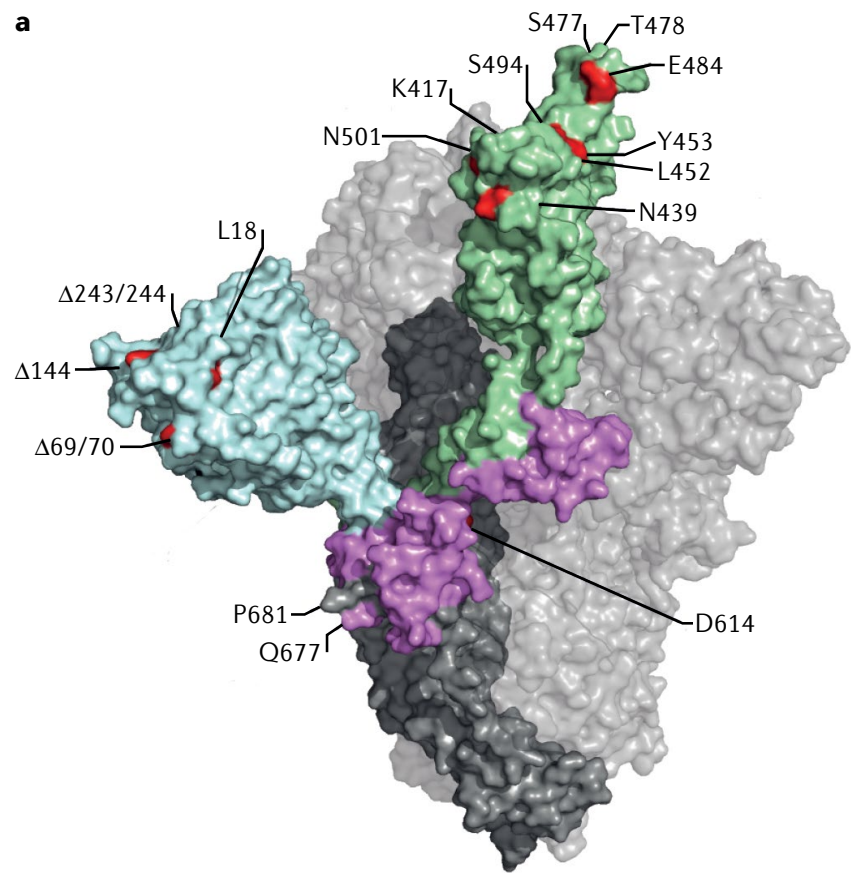

b
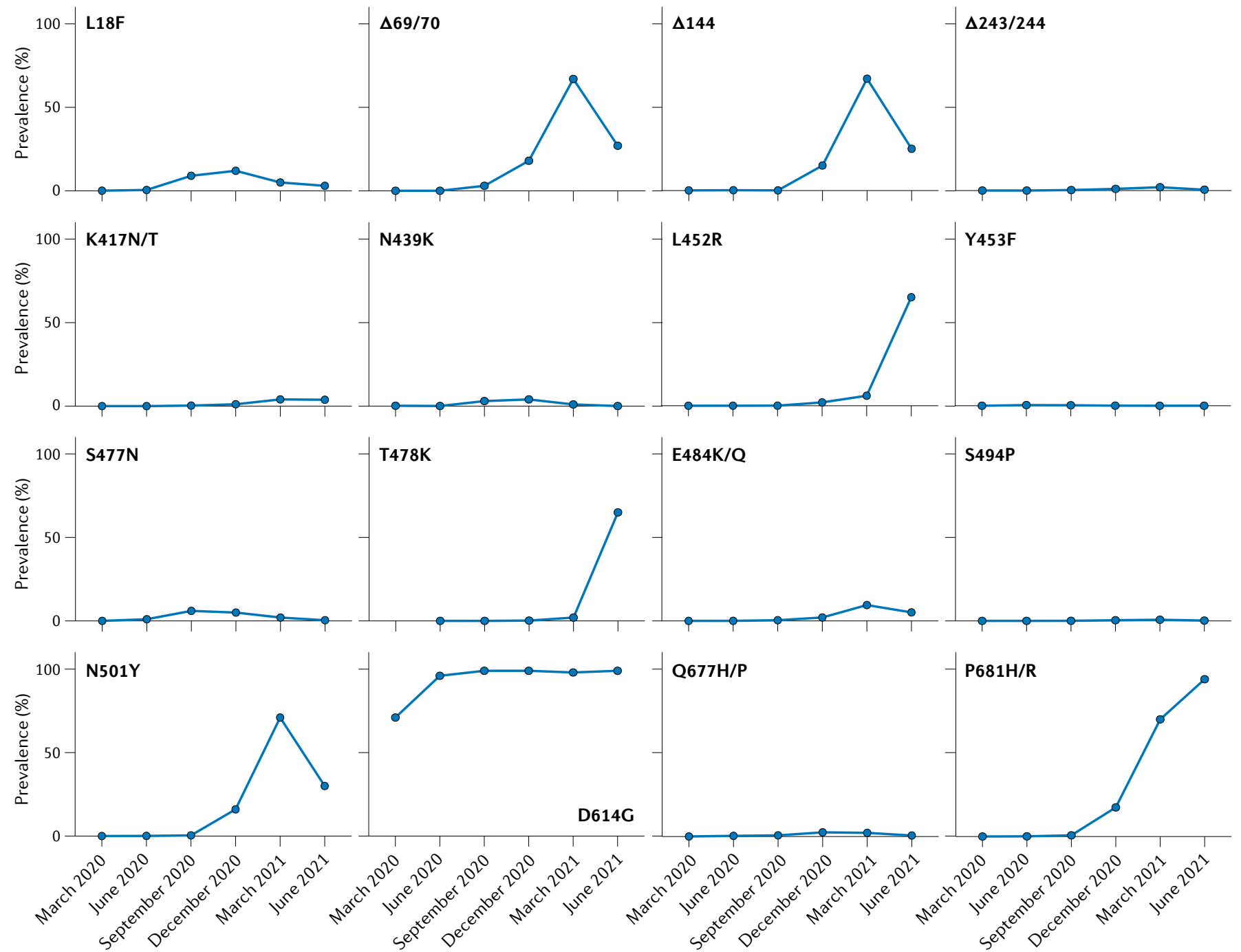
4 Fig. 4 | Locations and prevalence of key SARS-CoV-2 spike mutations. a | Sites of 16 key S1 (exposed attachment domain) mutations on the SARS-CoV-2 spike trimer, including 9 in the receptor-binding domain (RBD; green), four in the amino-terminal domain (NTD; cyan) and three in the carboxy-terminal domain (CTD; purple). Spike trimer figure derived from a cryo-electron microscopy structure (Protein Databank (PDB) code 7BNN). S2 (partially buried fusion domain) shown in dark grey. Six of the RBD mutations (K417N/T, L452R, T478K, E484K and N501Y) are present in one or more of the World Health Organization (WHO) variants of concern (VOCs): Alpha (B.1.1.7), Beta (B.1.351), Gamma (P.1), and Delta (B.1.617.2). NTD mutations include three deletions and L18F, mutations present in two or more VOCs. CTD mutations include D614G, which became the consensus amino acid at this position prior to the emergence of the VOCs, and $\mathrm{P} 681 \mathrm{H}$ and P681R, which are present in Alpha and Delta VOCs, respectively. $\mathbf{b}$ |Other than D614G, which has a prevalence close to $100 \%$, the most prevalent mutations as of June 2021 are those present in Alpha $(\Delta 69 / 70, \Delta 144, N 501 \mathrm{Y}$ and P681H) and Delta (L452R, T478K, and P681R) VOCs. Prevalence data obtained from outbreak.info ${ }^{196}$.

Transcription regulatory sequence

(TRS). In coronavirus genomes, a short sequence located at several genomic locations that is responsible for producing a set of nested $3^{\prime}$ and $5^{\prime}$

co-terminal sub-genomic RNA molecules.

Effective reproduction rate The average number of new infections caused by an infectious individual in a population where some individuals may no longer be susceptible. The effective reproduction rate of different variants is compared to control for factors other than transmissibility that might account for changes in the prevalence of different variants.

Secondary attack rate

The transmission of infection in a circumscribed group. The

secondary attack of different variants is compared to control for factors other than transmissibility that might account for changes in the prevalence of different variants. plasma $^{50,148,169}$. Increases in the prevalence of two lineages containing $\mathrm{N} 439 \mathrm{~K}$ were reported in the UK in September 2020, but their prevalence declined with the emergence of the Alpha variant (REF. ${ }^{169}$ ).

Y453F emerged independently several times in mink lineages, including one that subsequently spread among humans but is no longer active ${ }^{170}$. Y453F increases ACE2 binding but, nonetheless, remains rare ${ }^{128,140,171}$. Y453F markedly reduces susceptibility to casirivimab but not to the other FDA EUA-approved mAbs ${ }^{105,167}$ (TABLE 1).

S477N was present in a variant that spread widely in Europe in the summer of 2020 (REF. ${ }^{172}$ ). It increases the strength of ACE2 binding ${ }^{128}$ but has since circulated at a low level. It has not been shown to reduce susceptibility to any of the FDA EUA-approved mAbs ${ }^{109,167}$.

T478K is present in the Delta variant and a common variant in Mexico $^{34,173}$. By itself, it retains susceptibility to all but a few mAbs and to most convalescent and vaccinee plasma samples ${ }^{50,129,130,174}$.

F490S and S494P are uncommon RBM mutations that have arisen independently within several Alpha variant sub-lineages ${ }^{158}$. F490S is associated with highly reduced susceptibility to bamlanivimab but retains susceptibility to the other FDA EUA-approved mAbs ${ }^{64,105,175}$. S494P is associated with $>10$-fold reduced susceptibility to bamlanivimab and about 5-fold reduced susceptibility to casirivimab ${ }^{64,109,175}$.

\section{NTD mutations}

NTD deletions are present in several VOCs and VOIs, and have also been reported commonly in persons with prolonged SARS-CoV-2 infections ${ }^{112-114,176}$. Deletions at positions 69-70 appear to be associated primarily with increased virus replication ${ }^{113,177}$ whereas those between positions 141-146 and 242-244 interfere with the neutralizing activity of NTD-binding antibodies ${ }^{51,121,178,179}$. Other NTD mutations including L18F and D253Y also reduce susceptibility to NTD-neutralizing antibodies ${ }^{51,103,158}$.

\section{Mutations close to the S1/S2 furin cleavage site}

Mutations just upstream of the polybasic S1/S2 furin cleavage - including Q675H/R, Q677H/P, N679K and $\mathrm{P} 681 \mathrm{H} / \mathrm{R}$ - have occurred independently in many SARS-CoV-2 variants ${ }^{180}$. $\mathrm{P} 681 \mathrm{H}$ is present in the Alpha VOC and the Theta VOI, and in several additional SARS-CoV-2 lineages ${ }^{156}$. P681R is found in the Delta VOC and the Kappa VOI. The increased positive charge associated with both $\mathrm{P} 681 \mathrm{H}$ and $\mathrm{P} 681 \mathrm{R}$ appears to influence virus tropism by increasing $\mathrm{S} 1 / \mathrm{S} 2$ cleavage in human airway epithelial cells ${ }^{181-183}$.

\section{Non-spike mutations}

Mutations outside the spike protein have been reported to increase SARS-CoV-2 transmissibility by antagonizing the host response to type I interferons. In one study, the Alpha and Beta variants displayed a mean 112-fold and 8-fold reduced susceptibility to several type I interferons, respectively, compared with an early pandemic virus $^{184}$. In a second study, the Alpha variant was found to cause lower levels of interferon- $\beta$ (IFN $\beta$ ) expression and to be less sensitive to IFN $\beta$ pretreatment compared with two early pandemic viruses ${ }^{185}$. In this and in a third study, a D3L mutation in the Alpha variant nucleocapsid gene was found to introduce an enhanced transcription regulatory sequence (TRS) upstream of Orf $9 b$, an interferon antagonist gene expressed as an alternative reading frame within the nucleocapsid coding region ${ }^{185-187}$.

Although the nucleocapsid D3L mutation is not found in other VOCs or VOIs, most have mutations in genes associated with interferon antagonism. One example is a recurrent deletion $(\Delta 106-108)$ of unknown phenotypic consequence in nsp6, a component of the SARS-CoV-2 membrane-tethered complex that also antagonizes interferon ${ }^{188}$ and is present in the Alpha, Beta and Gamma VOCs and the Eta, Iota and Lambda VOIs $^{25}$ (FIG. 1 b). Another example is a recurrent adjacent three-nucleotide change in the nucleocapsid gene that probably arose by homologous recombination of the core sequence of the leader TRS and that results in the double amino acid change R203K/G204R and novel sub-genomic transcripts of unknown consequences ${ }^{189,190}$.

\section{SARS-CoV-2 variants}

SARS-CoV-2 variants are classified according to their lineage and component mutations. As a result, viruses belonging to the same lineage but containing different subsets of mutations can be classified as different variants. Variants are characterized by their transmissibility, disease severity and ability to evade humoral immunity. Increased transmissibility is demonstrated by the ability of a variant to outcompete other variants and to display a higher effective reproduction rate and/or secondary attack rate compared with other circulating variants ${ }^{8,191-193}$. Disease severity has been assessed using mortality data and rates of hospitalization ${ }^{194,195}$. Variants associated with higher virus levels may be more transmissible and/or cause more severe disease. Evasion of humoral immunity has been assessed by comparing a variant's susceptibility to mAbs, convalescent plasma and vaccinee plasma with that of other variants ${ }^{52,97}$. In the following sections, we summarize the biological, epidemiological and clinical characteristics of the WHO-defined VOCs and VOIs as of June 2021.

\section{Alpha variant (B.1.1.7)}

The Alpha variant spike mutations include the RBD mutation N501Y, P681H and NTD deletions at positions 69-70 and 144 (FIG. 1 b). The position 69-70 deletion prevents the amplification of one of three genomic segments 
Table 1 | SARS-CoV-2 variants and their fold reduction in neutralization susceptibility to monoclonal antibodies in advanced clinical trials

\begin{tabular}{|c|c|c|c|c|c|c|c|c|c|c|c|c|c|c|c|}
\hline Variant & BAM & ETE & BAM + ETE & CAS & IMD & CAS + IMD & SOT & REG & TIX & CIL & C144 & C135 & $\begin{array}{l}\text { BRII- } \\
196\end{array}$ & $\begin{array}{l}\text { BRII- } \\
198\end{array}$ & ADG20 \\
\hline $\begin{array}{l}\text { Alpha } \\
\text { (B.1.1.7) }\end{array}$ & $1_{12}$ & $3.6_{9}$ & $1.3_{3}$ & $1_{14}$ & $0.8_{15}$ & $1_{8}$ & $2.7_{12}^{a}$ & 1.4 & $1.5_{5}$ & $0.7_{5}$ & - & $0.9_{2}$ & $0.5_{3}$ & $0.2_{3}$ & 1.4 \\
\hline $\begin{array}{l}\text { Beta } \\
\text { (B.1.351) }\end{array}$ & $>100_{9}$ & $>100_{7}$ & $>100_{4}$ & $59_{11}$ & $0.8_{12}$ & $1.2_{8}$ & $1_{9}$ & $27_{2}$ & $4.9_{4}$ & $0.7_{4}$ & - & $0.9_{2}$ & $0.6_{5}$ & $6_{3}$ & 2.5 \\
\hline $\begin{array}{l}\text { Gamma } \\
\text { (P.1) }\end{array}$ & $>100_{2}$ & $59_{2}$ & $>100$ & $>100_{6}$ & $0.6_{6}$ & $1_{4}$ & $1_{6}$ & - & $6.2_{2}$ & $0.5_{2}$ & - & - & 0.3 & 0.7 & 2.2 \\
\hline $\begin{array}{l}\text { Delta } \\
\text { (B.1.617.2) }\end{array}$ & $>100_{2}$ & $0.7_{2}$ & - & $0.8_{2}$ & $0.8_{2}$ & 1 & - & - & 0.5 & 2.9 & - & - & - & - & 1.4 \\
\hline $\begin{array}{l}\text { Карра } \\
\text { (B.1.617.1) }\end{array}$ & $>100_{2}$ & $0.6_{2}$ & 5 & $4.6_{2}$ & $1.3_{2}$ & $1_{2}$ & 0.7 & - & 0.7 & 5.1 & - & - & - & - & 2.5 \\
\hline $\begin{array}{l}\text { Epsilon } \\
\text { (B.1.427/9) }\end{array}$ & $>100_{2}$ & $3.3_{2}$ & 7.7 & 1.3 & 2.1 & 1 & $0.8_{2}$ & 14 & - & - & - & - & - & - & - \\
\hline N501Y & $1_{3}$ & $2.9_{7}$ & 1 & $1_{9}$ & $0.8_{9}$ & $1_{3}$ & $1.6_{5}{ }^{a}$ & - & $1.1_{3}$ & $1_{3}$ & $1.4_{3}$ & $1.4_{3}$ & $1_{3}$ & $1.8_{2}$ & - \\
\hline E484K & $>100_{4}$ & $2.7_{6}$ & 17 & $13_{12}$ & $1_{12}$ & $1.6_{6}$ & $0.6_{5}$ & - & $6.4_{4}$ & $1.5_{4}$ & $>100_{4}$ & $0.4_{3}$ & $1.4_{3}$ & $2.4_{2}$ & - \\
\hline $\mathrm{K} 417 \mathrm{~N}$ & $0.2_{2}$ & $>100_{5}$ & 1 & $8.9_{7}$ & $0.9_{6}$ & 0.8 & $0.6_{4}$ & - & $0.3_{3}$ & $0.6_{3}$ & $0.7_{2}$ & $0.3_{2}$ & $1.8_{3}$ & $0.3_{2}$ & - \\
\hline L452R & $>100_{2}$ & $0.9_{4}$ & 7.4 & $1.2_{4}$ & $2_{4}$ & $1.2_{3}$ & 0.6 & - & - & - & - & - & 1.4 & - & - \\
\hline T478K & - & - & - & - & - & - & - & - & - & - & 1.5 & - & - & - & - \\
\hline N439K & 1.3 & $0.4_{3}$ & - & $0.8_{4}$ & $28_{5}$ & 1.7 & $0.9_{3}$ & - & - & - & $0.9_{2}$ & $>100$ & $1_{2}$ & 1 & - \\
\hline Y453F & 1.8 & $1.4_{3}$ & - & $74_{6}$ & $1.6_{6}$ & 3.5 & 1.1 & - & - & - & 1.1 & - & 1 & 1.5 & - \\
\hline F490S & $>100_{2}$ & $1.1_{2}$ & 1 & $1_{2}$ & $1.4_{2}$ & 1 & $0.8_{2}$ & - & - & - & $4_{2}$ & 1.2 & - & - & - \\
\hline S494P & $86_{2}$ & $0.6_{2}$ & 1 & $4.5_{3}$ & $0.9_{2}$ & 1.1 & $2_{2}$ & - & - & - & $73_{2}$ & 0.8 & 0.7 & 1.6 & - \\
\hline
\end{tabular}

Table shows fold reductions in neutralization (relative to control virus) for pseudo-typed and infectious viruses with combinations of spike mutations present in four World Health Organization (WHO)-defined variants of concern (VOCs; Alpha, Beta, Gamma and Delta), two WHO-defined variants of interest (VOls;

Kappa and Epsilon) and viruses containing individual spike mutations. Fold change is the median value of results, subscript is the number of results. '-' indicates absence of susceptibility data. 'Levels of fold reduction for SOT for N501Y and B.1.1.7 were much higher for IC values than for IC $_{50}$ values. BAM, bamlanivimab (LY-CoV555); CAS, casirivimab (REGN10933); CIL, cilgavimab (AZD1061); ETE, etesevimab (LY-CoV016); IMD, imdevimab (REGN10987); REG, regdanvimab (CT-P59); SOT, sotrovimab (Vir-7831); TIX, Tixagevimab (AZD8895).

in a commonly used diagnostic PCR assay, resulting in a phenomenon referred to as S-gene target failure (SGTF), which has been used as a proxy for this variant ${ }^{191}$. The Alpha variant also contains several non-spike mutations including nsp6: $\Delta 106-108$ and the nucleocapsid mutations D3L, R203K and G204R, which may increase transmission by antagonizing innate immunity ${ }^{25,185-187}$. By the second quarter of 2021, the Alpha variant accounted for the majority of infections in the USA and many European countries ${ }^{23,196}$. Epidemiological studies suggest that it was approximately $50 \%$ more transmissible than previously circulating UK variants ${ }^{191,197,198}$. It was also associated with threefold to eightfold higher upper-airway levels ${ }^{199-201}$ and an estimated 50\% increased mortality ${ }^{194,195,202}$.

The Alpha variant is susceptible to neutralization by most neutralizing mAbs as well as by most plasma samples from previously infected persons $s^{97,102,121,149,159,203-206}$ (FIG. 5). The fact that the Alpha variant is rarely associated with reduced susceptibility to convalescent plasma is consistent with it not being associated with an increased risk of reinfection ${ }^{207}$.

The Alpha variant has displayed 3-fold to 10 -fold reduced susceptibility to approximately $15 \%$ of plasma samples from recipients of an authorized mRNA vaccine $^{97,102,121,148,149,152,159,204,208,209}$. In cohort studies from Israel and Qatar, the BNT162b vaccine also retained greater than $90 \%$ efficacy against this variant ${ }^{210,211}$
(TABLE 2). In a post-hoc analysis of a NVX-CoV2373 clinical trial, vaccine efficacy was $86.3 \%$ against Alpha variants compared with $96.4 \%$ against non-Alpha variants ${ }^{212}$ (TABLE 2). Similar data for the AZD1222 vaccine have been inconsistent. In a study of vaccine trial participants, plasma samples were associated with a median reduction in neutralizing activity of ninefold to the Alpha variant compared with an earlier UK variant ${ }^{213}$. In this trial, AZD1222 displayed a non-statistically significant reduction in efficacy against the Alpha variant (70\%; 95\% confidence interval (CI) 44-85\%) compared with earlier variants $(82 \% \text {; } 95 \% \text { CI } 68-89 \%)^{213}$. However, in three other studies, the median reduction in neutralizing activity was between onefold and threefold ${ }^{102,214,215}$.

Several Alpha variant sub-lineages have acquired additional mutations that might increase the risk of reinfection and vaccine failure, including the RBD mutations E484K, F490S and S494P (REF. ${ }^{158}$ ).

\section{Beta variant (B.1.351)}

Between October 2020 and January 2021, daily cases in South Africa increased from approximately 2,000 to more than 20,000 reported cases per day. This increase occurred in a setting in which more than $30 \%$ of the population was estimated to have already been infected and was associated with the emergence of the Beta variant, which contains three RBD mutations (K417N, E484K and N501Y) and five NTD mutations, 

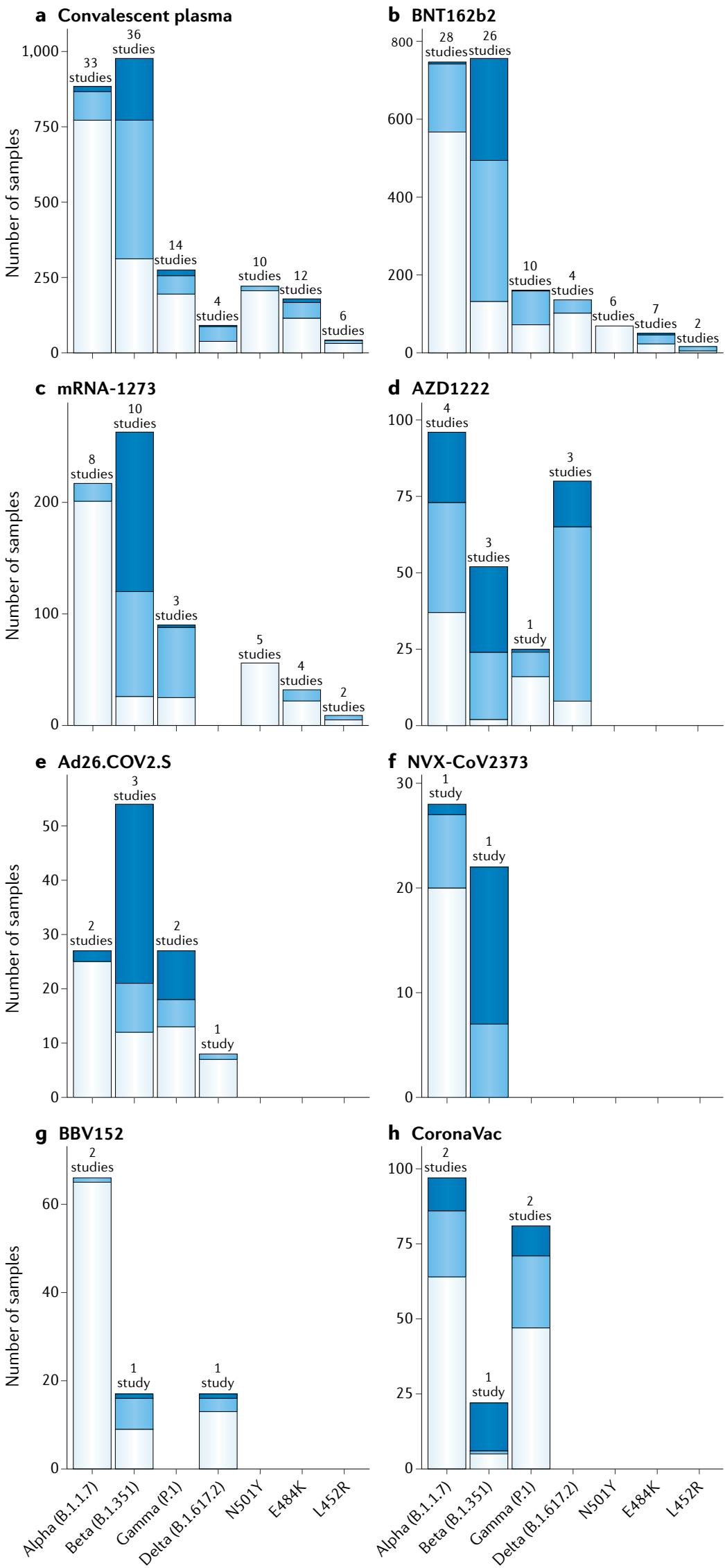

$<3$-fold reduced susceptibilty 3-fold to 10-fold reduced susceptibilty $>10$-fold reduced susceptibilty
Fig. 5 | Effects of SARS-CoV-2 spike variants on susceptibility to neutralization. Fold-reduced susceptibility of the four variants of concern (VOCs; Alpha, Beta, Gamma and Delta) and three common spike receptor-binding domain (RBD) mutations (N501Y, E484K and $(452 R$ ) to in vitro neutralization by plasma from previously infected persons (part a) and from persons vaccinated with the Pfizer/BioNTech BNT162b2 (part b), Moderna mRNA-1273 (part c), AstraZeneca AZD1222 (part d), Janssen Ad26.COV2.S (part e), Novavax NVXCoV2373 (part f), Bharat Biotech BBV152 (part g) and Sinovac CoronaVac (part $\mathbf{h}$ ) vaccines. y axes indicate number of plasma units tested. Colour scheme indicates fold reduction in neutralization. Only those data from plasma samples from persons receiving a full immunization schedule were included. Data obtained from https:// covdb.stanford.edu/search-drdb/ on 1 July 2021. In some plots, distributions are approximate as they include results reported only in aggregate as a mean fold reduction in susceptibility.

including a deletion within the NTD supersite at positions 242-244 (REF. ${ }^{16}$ ). The Beta variant was estimated to be $50 \%$ more transmissible than the lineages that preceded $\mathrm{it}^{16}$.

Reinfections with the Beta variant occurred commonly during a phase IIb trial of the NVX-CoV2373 vaccine performed in South Africa as approximately one third of infections in both the vaccine and placebo arms were reinfections ${ }^{216}$. It is not known whether the Beta variant is associated with higher virus levels or disease severity because once detected it was no longer co-circulating with other lineages. As of June 2021, the Beta variant accounts for more than $50 \%$ of infections in many countries in sub-Saharan Africa ${ }^{23,196}$.

The Beta variant is associated with reduced susceptibility to many mAbs because $\mathrm{E} 484 \mathrm{~K}$ interferes with binding of several RBM class 1 and $2 \mathrm{mAbs}$ and $\mathrm{K} 417 \mathrm{~N}$ interferes with the binding of several RBM class 1 $\mathrm{mAbs}^{104,117,121,163,217}$. Of the five FDA EUA-approved mAbs, bamlanivimab, etesevimab and casirivimab are largely inactive against B.1.351 whereas imdevimab and sotrovimab, which bind to the RBD core, retain neutralizing activity ${ }^{104,109,117,175,204}$ (TABLE 1).

The Beta variant has displayed 3-fold to 10 -fold reduced susceptibility to $46 \%$ and $>10$-fold reduced susceptibility to $22 \%$ of convalescent plasma samples compared with early pandemic variants ${ }^{97,101,104,121,124,203,204,218-220}$ (FIG. 5). Of 34 convalescent plasma samples from persons infected with the Alpha variant, 59\% had 3-fold to 10 -fold reduced neutralizing activity and $18 \%$ had $>10$-fold reduced neutralizing activity to the Beta variant $^{99,104,221}$. Conversely, plasma from 22 persons infected with the Beta variant retained partial or full neutralizing activity against variants from earlier waves of the pandemic in all but three persons ${ }^{101}$.

Of plasma samples obtained from persons receiving one of the mRNA vaccines, $45 \%$ had 3 -fold to 10 -fold and $30 \%$ had $>10$-fold reduced Beta variant neutralizing activity $97,104,117,121,151,204,208,209,222,223$ (FIG. 5). Of plasma samples from persons receiving the AZD1222 vaccine, $42 \%$ had 3 -fold to 10 -fold and $54 \%$ had $>10$-fold reduced Beta variant neutralizing activity ${ }^{104,214,224}$ (FIG. 5). 
Table 2 | Vaccine efficacy at preventing symptomatic infection for SARS-CoV-2 variants of concern

\begin{tabular}{|c|c|c|c|c|c|}
\hline Variant & $\begin{array}{l}\text { Pfizer/BioNTech } \\
\text { BNT162b }\end{array}$ & $\begin{array}{l}\text { AstraZeneca } \\
\text { AZD1222 }^{\mathrm{a}}\end{array}$ & $\begin{array}{l}\text { Novavax NVX- } \\
2373\end{array}$ & $\begin{array}{l}\text { Janssen } \\
\text { AD26.CoV2 }\end{array}$ & $\begin{array}{l}\text { Sinovac } \\
\text { CoronaVac }\end{array}$ \\
\hline Pre-variant & $95 \%(\text { trial) })^{5,59}$ & $70 \%$ (trial) $^{59,248}$ & $96 \%(\text { trial) })^{59,212}$ & $72 \%$ (trial) $^{59,225}$ & $51 \%(\text { trial })^{59}$ \\
\hline \multirow[t]{4}{*}{ Alpha } & $90 \%(\text { TNCC })^{211}$ & $70 \%(\text { trial) })^{213}$ & \multirow[t]{4}{*}{$86 \%\left(\right.$ trial) ${ }^{212}$} & \multirow[t]{4}{*}{-} & \multirow[t]{4}{*}{-} \\
\hline & $92 \%(\text { TNCC })^{233}$ & $66 \%(\text { TNCC })^{232}$ & & & \\
\hline & $93 \%(\text { TNCC })^{232}$ & \multirow[t]{2}{*}{$81 \%(\text { TNCC })^{233}$} & & & \\
\hline & $97 \%$ (cohort $^{210}$ & & & & \\
\hline Beta & $75 \%(\text { TNCC })^{211}$ & $10 \%$ (trial) $^{224}$ & $51 \%(\text { trial) })^{216}$ & $64 \%\left(\right.$ trial) ${ }^{225}$ & - \\
\hline Gamma & - & - & - & - & $42 \%(\text { TNCC })^{249}$ \\
\hline \multirow[t]{2}{*}{ Delta } & $83 \%(\text { TNCC })^{233}$ & $60 \%(\text { TNCC })^{232}$ & \multirow[t]{2}{*}{-} & \multirow[t]{2}{*}{-} & \multirow[t]{2}{*}{-} \\
\hline & $88 \%(\text { TNCC })^{232}$ & $61 \%(\text { TNCC })^{233}$ & & & \\
\hline
\end{tabular}

Table shows vaccine efficacy at preventing symptomatic SARS-CoV-2 infection with the Alpha, Beta, Gamma and Delta variants of concern (VOCs). Comparison is with efficacy against 'pre-variant' isolates. Data include persons receiving a full course of vaccination: 1-2 weeks following two vaccine doses for BNT162b, AZD1222, NVX-2373 and CoronaVac; 4 weeks after one vaccine dose for AD26.CoV2. 'Trial' refers to post-hoc analysis of clinical trial data. TNCC, test-negative case-control study; '-', Not available. ${ }^{a}$ AZD 1222 efficacy data include some patients receiving the standard vaccine dose at both time points and others receiving a lower vaccine dose at the first time point. ${ }^{\mathrm{b}} \mathrm{AD} 26 . \mathrm{CoV} 2$ efficacy data based on preventing moderate to severe disease; pre-variant efficacy based on US data. ${ }^{c}$ CoronaVac efficacy data against pre-variant isolates may include some persons infected with the Gamma VOC and the Zeta variant of interest (VOI). The TNCC study of vaccine efficacy against the Gamma VOC was performed in persons aged 70 years or older.

The Beta variant has also been associated with reduced vaccine efficacy (TABLE 2). In a phase II trial in South Africa of AZD1222 in which 39 HIV-negative patients became infected with the Beta variant, the estimated vaccine efficacy was just $10 \%{ }^{224}$. In a phase II trial of NVX-CoV2373 in South Africa in which 38 of 41 infections were caused by the Beta variant, the estimated vaccine efficacy was $49 \%{ }^{216}$. In a phase III trial of the Janssen Ad26.COV2.S vaccine, vaccine efficacy in South Africa was 52\% and 64\% against moderate to severe COVID-19 with onset at least 14 and 28 days after administration, respectively ${ }^{225}$. In the previously cited Qatar cohort study, the efficacy of BNT162b against the Beta variant was estimated to be $72 \%$, which was $15 \%$ lower than that for the Alpha variant ${ }^{211}$. Among nine BNT162b vaccine breakthrough infections in an Israeli case-control study, eight were with the Beta variant even though the Alpha variant predominated during the study period ${ }^{226}$.

\section{Gamma variant (P.1)}

The Gamma variant contains the RBD mutations N501Y, E484K and K417T (REF. ${ }^{19}$ ). It also contains five NTD mutations, of which L18F has been shown to interfere with the binding of NTD-targeting neutralizing antibodies $^{51}$. As the Gamma variant was associated with a surge of infections in a region of Brazil estimated to have achieved a high infection rate, it is suspected of being able to infect and cause illness in persons previously infected with other variants ${ }^{19,29,30}$. It was estimated to result in virus levels 3-4 times higher than earlier variants and to be responsible for an estimated 1.1-fold to 1.8 -fold higher mortality ${ }^{19}$. By June 2021, the Gamma variant accounted for a high proportion of infections in several South American and Caribbean countries ${ }^{196}$ and $10 \%$ of US infections ${ }^{33}$. The resistance profile of the Gamma variant to the FDA EUA-approved mAbs is similar to that of the Beta variant ${ }^{103,109,217,227,228}$ (TABLE 1). Of convalescent plasma samples obtained from persons infected with early pandemic variants or with the Alpha variant, about $20 \%$ had 3 -fold to 10 -fold and $10 \%$ had $>10$-fold reduced neutralizing activity ${ }^{97,103,117,203,217,228,229}$ (FIG. 5). Of plasma samples from persons receiving one of the two authorized mRNA vaccines, about $60 \%$ had 3 -fold to 10 -fold and $5 \%$ had $>10$-fold reduced neutralizing activity $97,103,117,167,217,230$ (FIG. 5). A similar distribution in the reduction in neutralizing activity was observed in plasma samples from recipients of the AZD1222 vaccine ${ }^{103}$.

\section{Delta variant (B.1.617.2)}

As the pandemic surged in India in early 2021, two variants sharing a common ancestor, Delta (B.1.617.2) and Kappa (B.1.617.1), accounted for a high proportion of infections. The two variants probably diverged from a common ancestor between August and October 2020 (FIG. 1). Both variants contain the RBD mutation L452R, the proximal furin cleavage site mutation P681R and several mutations within orf3, orf7a and the nucleocapsid gene. The Kappa variant contained the RBD mutation E484Q whereas the Delta variant contained the RBD mutation T478K. The two variants also contain different mutations within orf $1 a / b$ and the spike NTD and S2 domains. Even though E484Q is more likely than T478K to evade antibody neutralization ${ }^{48,100,165}$, only the Delta variant has demonstrated increased transmissibility, spreading to 54 countries and rapidly replacing the Alpha variant in the $\mathrm{UK}^{32,35}$ and the $\mathrm{USA}^{33}$.

Among the FDA EUA-approved mAbs, the Delta variant is associated with high-level reduced bamlanivimab susceptibility ${ }^{100,214}$. It results in approximately 3 -fold to 10 -fold reduced susceptibility to $45 \%$ and $>10$-fold reduced susceptibility to $5 \%$ of convalescent plasma samples ${ }^{100,118,214,215,231}$ (FIG. 5). Among plasma samples obtained from recipients of the BNT162b vaccine, approximately $15 \%$ displayed 3 -fold to 10 -fold reduced neutralizing activity against the Delta variant ${ }^{100,118,214}$ (FIG. 5). 
By contrast, among plasma samples obtained from recipients of the AZD1222 vaccine, higher proportions displayed reduced neutralizing activity against this variant ${ }^{100,209,214}$. In case-control studies from the UK, the BNT162b vaccine was approximately $85 \%$ effective for the Delta variant whereas the AZD1222 vaccine was approximately $60 \%$ effective $\mathrm{e}^{232,233}$.

AY. 1 and AY.2 are recently reported sub-lineages of the Delta variant that have developed additional mutations including the RBD mutation K417N (REF. ${ }^{196}$ ).

\section{Variants of interest}

The Epsilon variant comprises two closely related lineages B.1.427 and B.1.429, first detected in California, USA. This variant was the first reported to contain the RBD mutation L452R. It was estimated to be about $20 \%$ more transmissible than co-circulating lineages and to be associated with twofold higher upper-airway virus levels ${ }^{123,192}$. By February 2021, the Epsilon variant accounted for $15 \%$ of US infections, but by June 2021 its prevalence decreased to below $1 \%$.

In contrast to the Delta VOC, the Kappa VOI has not demonstrated increased transmissibility. However, because of the presence of the RBD mutations L452R and $\mathrm{E} 484 \mathrm{Q}$, the Kappa variant has a somewhat greater ability to evade humoral immunity than the Delta variant. It displays reduced susceptibility to casirivimab and to bamlanivimab. Of convalescent plasma samples, about $40 \%$ had 3 -fold to 10 -fold and $15 \%$ had $>10$-fold reduced neutralizing activity ${ }^{100,122,234}$. Of plasma samples from recipients of an mRNA vaccine, approximately $55 \%$ had 3 -fold to 10 -fold and $5 \%$ had $>10$-fold reduced neutralizing activity ${ }^{100,118,122,234}$.

The Iota (B.1.526), Eta (B.1.525) and Zeta (P.2) variants are each characterized primarily by the RBD mutation E484K. The Iota variant was first identified in New York state. In June 2021, it had a prevalence of 5-10\% in the USA but remained rare outside the USA ${ }^{154}$. It contains the same nsp6 deletion as in the Alpha, Beta and Gamma variants. Of convalescent plasma samples, about $40 \%$ display 3 -fold to 10 -fold and $10 \%$ display $>10$-fold reduced Iota variant neutralizing activity ${ }^{166,235}$. Of plasma samples from recipients of an mRNA vaccine, about $30 \%$ display 3 -fold to 10 -fold reduced neutralizing activity $^{230,235}$. The Eta variant is present at low levels in many countries, with Nigeria having the highest proportion of infections ${ }^{236}$. The ability of $\mathrm{mAb}$, convalescent plasma and vaccinee plasma to neutralize the Eta variant has been infrequently studied ${ }^{118,166}$. The Zeta variant was common in Brazil in late 2020 and early 2021 (REF. ${ }^{157}$ ), but appears to be decreasing in prevalence.

The Theta variant was first reported in March 2021 in the Philippines. It contains 13 lineage-defining mutations including N501Y, E484K, P681H and the NTD deletion at positions $141-143$ (REFS $^{155,156}$ ). However, it remains rare, accounting for a small proportion of infections even in the Philippines ${ }^{196}$. The Lambda variant (C.37) has a unique set of spike mutations including L452Q and F490S within the RBD and the NTD deletion $\triangle 246-252\left(\right.$ REF. $\left.^{237}\right)$. It is highly prevalent in several South American countries and is associated with reduced susceptibility to the locally used CoronaVac vaccine ${ }^{238}$.

\section{Conclusions and implications for COVID-19}

SARS-CoV-2 variants are characterized by their transmissibility, disease severity and ability to evade humoral immunity. The Alpha and Delta variants are each associated with increased transmissibility and greater disease severity because of immune evasion, and potentially because of higher virus levels resulting from the antagonism of innate immunity. The Beta and Gamma variants are each associated with increased transmissibility because of their ability to evade humoral immunity and cause reinfections. Although it is not surprising that mutations associated with reduced humoral immunity have recently emerged in association with rising population immunity, the concurrent emergence of mutations that intrinsically increase SARS-CoV-2 replication is more difficult to explain. The timing of this second category of mutations raises the possibility that they only emerged after a critical number of global infections or as compensation for subtle reductions in replication fitness associated with developing immune escape mutations.

As of June 2021, it is uncertain whether the current approaches to classifying variants will be sustainable. Should the current VOCs and VOIs develop multiple sub-lineages with additional biologically relevant mutations, it may become necessary to classify variants according to their component mutations rather than their ancestral lineages. However, despite the phenomenal progress in studying the impact of individual mutations in vitro and in animal models, classifying variants according to their component mutations will also prove challenging should the number of recurrent mutations also increase.

Although SARS-CoV-2 variants differ in their transmission rates, disease severity and risk of reinfection, there is no evidence that they are differentially affected by non-pharmaceutical public health measures such as social distancing and the use of personal protective equipment, or that they will respond differently to most antiviral therapies. Except for several $\mathrm{mAb}$ preparations (for example, bamlanivimab/etesevimab), most antiviral compounds are likely to retain activity against each of the current VOCs and VOIs. The two most recently approved $\mathrm{mAb}$ preparations, casirivimab/imdevimab and sotrovimab, retain activity against all VOCs and VOIs, in part because imdevimab and sotrovimab target the RBD core whereas the most prevalent immune escape mutations are in the RBD RBM. There have also been no reports of variants with mutations in the enzymatic targets of therapy that would reduce susceptibility to remdesivir or to the RNA-dependent RNA polymerase and protease inhibitors in clinical development.

The most important consequence of emergent SARS-CoV-2 variants, therefore, is their impact on vaccine efficacy. The levels of neutralizing mAbs elicited by the mRNA vaccines has been high and in most persons are likely to be maintained for many months above the levels required for protection against even the most resistant circulating variants ${ }^{164,239,240}$. In addition, the mRNA vaccines appear to often elicit memory B cells that undergo somatic hypermutation, which should broaden the response to viruses with variant spike 
proteins ${ }^{239,240}$. Nonetheless, in vitro neutralization studies and epidemiological vaccine efficacy studies indicate weaker protection against emerging variants for most of the non-mRNA vaccines. Moreover, regardless of the vaccine received, reductions in vaccine-elicited humoral immunity is likely to be clinically significant for persons with impaired immunity as a result of underlying disease, immunosuppressive drugs or older age $\mathrm{e}^{241-244}$.

As the spectrum of SARS-CoV-2 variants is expanding and shifting faster than epidemiological studies can be conducted, laboratory correlates of protection against SARS-CoV-2 variants have become a high priority. As summarized in this Review, neutralizing antibody titres against pre-variant and variant SARS-CoV-2 isolates correlate with protection from infection in epidemiological studies. Whether this is because neutralizing antibodies are the most important means of viral protection and/or because these titres correlate with other aspects of protective immunity, including memory B cells, antibody-mediated effector functions and T cell immunity is not known. Therefore, a strategy that combines genomic surveillance, in vitro neutralization studies and vaccine efficacy studies should be maintained to identify those variants that pose the greatest threat to current vaccines and to guide the development of immunogens for second-generation vaccines $^{245}$.

An mRNA vaccine that incorporated most of the spike mutations present in the Beta variant (mRNA1273.351; Moderna) was recently reported to increase both pre-variant and Beta variant-specific neutralizing antibody titres when administered as a booster to mice that had previously been immunized with mRNA-1273 $\left(\mathrm{REF}^{246}\right)$. Continued studies in animal species that are more predictive of responses in humans will be necessary to determine whether updated immunogens can broaden the response to multiple variants rather than just boost existing antibody responses generated by previous infections or vaccinations ${ }^{247}$. Moreover, as the spike protein has been found to contain multiple cytotoxic and helper T cell epitopes, it will be important that as many of these as possible are included in future vaccine preparations ${ }^{89}$.

Published online 17 September 2021
1. Minskaia, E. et al. Discovery of an RNA virus $3^{\prime} \rightarrow 5^{\prime}$ exoribonuclease that is critically involved in coronavirus RNA synthesis. Proc. Natl Acad. Sci. USA 103, 5108-5113 (2006).

2. Eckerle, L. D. et al. Infidelity of SARS-CoV Nsp14-exonuclease mutant virus replication is revealed by complete genome sequencing. PLoS Pathog. 6, e1000896 (2010).

3. Duffy, S., Shackelton, L. A. \& Holmes, E. C. Rates of evolutionary change in viruses: patterns and determinants. Nat. Rev. Genet. 9, 267-276 (2008).

4. Graham, R. L. \& Baric, R. S. Recombination, reservoirs, and the modular spike: mechanisms of coronavirus cross-species transmission. J. Virol. 84, 3134-3146 (2010).

5. Gribble, J. et al. The coronavirus proofreading exoribonuclease mediates extensive viral recombination. PLoS Pathog. 17, e 1009226 (2021).

6. Jackson, B. et al. Generation and transmission of inter-lineage recombinants in the SARS-CoV-2 pandemic. Preprint at medRxiv https://doi. org/10.1101/2021.06.18.21258689 (2021)

7. Boni, M. F. et al. Evolutionary origins of the SARS-CoV-2 sarbecovirus lineage responsible for the COVID-19 pandemic. Nat. Microbiol. 5, 1408-1417 (2020).

8. Korber, B. et al. Tracking changes in SARS-CoV-2 spike: evidence that D614G increases infectivity of the COVID-19 virus. Cell 182, 812-827.e19 (2020).

This paper is the first to show evidence of evolutionary selection pressure by demonstrating that a variant containing D614G independently outcompeted other variants in multiple geographical locations

9. Simmonds, P. Rampant $\mathrm{C} \rightarrow U$ hypermutation in the genomes of SARS-CoV-2 and other coronaviruses: causes and consequences for their short- and long-term evolutionary trajectories. $m S$ phere $\mathbf{5}$ e00408-e00420 (2020).

10. van Dorp, L. et al. No evidence for increased transmissibility from recurrent mutations in SARS-CoV-2. Nat. Commun. 11, 5986 (2020).

11. Edridge, A. W. D. et al. Seasonal coronavirus protective immunity is short-lasting. Nat. Med. 26, 1691-1693 (2020)

12. Kistler, K. E. \& Bedford, T. Evidence for adaptive evolution in the receptor-binding domain of seasonal coronaviruses OC43 and 229E. eLife 10, e64509 (2021).

13. Eguia, R. T. et al. A human coronavirus evolves antigenically to escape antibody immunity. PLoS Pathog. 17, e1009453 (2021). This paper presents a retrospective analysis of HCoV-229E sequences and plasma samples that shows that human coronaviruses can evolve over several years to resist antibody neutralization, providing a potential mechanism for recurrent human coronavirus infections.

14. Candido, D. S. et al. Evolution and epidemic spread of SARS-CoV-2 in Brazil. Science 369, 1255-1260 (2020).

15. Tegally, H. et al. Sixteen novel lineages of SARS-CoV-2 in South Africa. Nat. Med. 27, 440-446 (2021).

16. Tegally, H. et al. Detection of a SARS-CoV-2 variant of concern in South Africa. Nature 592, 438-443 (2021) This molecular epidemiological study charts the spread of the beta variant in South Africa and shows that it resulted from selective evolutionary forces.

17. Pereson, M. J. et al. Phylogenetic analysis of SARS-CoV-2 in the first few months since its emergence. J. Med. Virol. 93, 1722-1731 (2021).

18. Day, T., Gandon, S., Lion, S. \& Otto, S. P. On the evolutionary epidemiology of SARS-CoV-2. Curr. Biol. 30, R849-R857 (2020)

19. Faria, N. R. et al. Genomics and epidemiology of the P. 1 SARS-CoV-2 lineage in Manaus, Brazil. Science 372, 815-821 (2021).

This paper presents an analysis showing that the Gamma variant is about twofold more transmissible than previous variants in Manaus, Brazil, and that it poses an increased risk of reinfection compared with previous variants.

20. Morel, B. et al. Phylogenetic analysis of SARS-CoV-2 data is difficult. Mol. Biol. Evol. 38, 1777-1791 (2020).

21. Mavian, C. et al. Sampling bias and incorrect rooting make phylogenetic network tracing of SARS-COV-2 infections unreliable. Proc. Natl Acad. Sci. USA 117 12522-12523 (2020).

22. Rambaut, A. et al. A dynamic nomenclature proposal for SARS-CoV-2 lineages to assist genomic epidemiology. Nat. Microbiol. 5, 1403-1407 (2020).

23. Hodcroft, E. CoVariants: SARS-CoV-2 mutations and variants of interest (NextStrain). CoVariants https:/ covariants.org/ (2021)

24. Dearlove, B et al A SARS-CoV-2 vaccine candidate would likely match all currently circulating variants. Proc. Natl Acad. Sci. USA 117, 23652-23662 (2020).

25. Martin, D. P. et al. The emergence and ongoing convergent evolution of the N501Y lineages coincides with a major global shift in the SARS-CoV-2 selective landscape. Preprint at medRxiv https://doi.org/ 10.1101/2021.02.23.21252268 (2021).

26. MacLean, O. A. et al. Natural selection in the evolution of SARS-CoV-2 in bats created a generalist virus and highly capable human pathogen. PLOS Biol. 19, e3001115 (2021)

27. Rambaut, A. et al. Preliminary genomic characterisation of an emergent SARS-CoV-2 lineage in the UK defined by a novel set of spike mutations. Virological https://virological org/t/563 (2020).

28. Karim, S. S. A. \& de Oliveira, T. New SARS-CoV-2 variants - clinical, public health, and vaccine implications. N. Engl. J. Med. 384, 1866-1868 (2021).

29. Buss, L. F et al. Three-quarters attack rate of SARS-CoV-2 in the Brazilian Amazon during a largely unmitigated epidemic. Science 371, 288-292 (2021).

30. Sabino, E. C. et al. Resurgence of COVID-19 in Manaus, Brazil, despite high seroprevalence. Lancet 397, 452-455 (2021).

31. Salvatore, M. et al. Resurgence of SARS-CoV-2 in India: potential role of the B.1.617.2 (delta) variant and delayed interventions. Preprint at medRxiv https: doi.org/10.1101/2021.06.23.21259405 (2021).

32. Riley, S. et al. REACT-1 round 12 report: resurgence of SARS-CoV-2 infections in England associated with increased frequency of the Delta variant. Preprint at medRxiv https://doi.org/10.1101/ 2021.06.17.21259103 (2021).

33. Bolze, A. et al. Rapid displacement of SARS-CoV-2 variant B.1.1.7 by B. 1.617.2 and P.1 in the United States. Preprint at medRxiv https://doi.org/10.1101/ 2021.06.20.21259195 (2021).

34. Dhar, M. S. et al. Genomic characterization and epidemiology of an emerging SARS-CoV-2 variant in Delhi, India. Preprint at medRxiv https://doi.org/ 10.1101/2021.06.02.21258076 (2021).

35. Challen, R. et al. Early epidemiological signatures of novel SARS-CoV-2 variants: establishment of B.1617.2 in England. Preprint at medRxiv https:// doi.org/10.1101/2021.06.05.21258365 (2021).

36. Public Health England. SARS-CoV-2 variants of concern and variants under investigation in England. Public Health England https://assets.publishing. service.gov.uk/government/uploads/system/uploads/ attachment_data/file/993879/Variants_of_Concern_ VOC_Technical_Briefing_15.pdf (2021).

37. WHO. SARS-CoV-2 variants of concern and variants of interest. World Health Organization https://www.who. int/en/activities/tracking-SARS-CoV-2-variants/ (2021)

38. CDC. SARS-CoV-2 variant classifications and definitions. Centers for Disease Control and Prevention https://www.cdc.gov/coronavirus/ 2019-ncov/variants/variant-info.html (2021).

39. The COVID-19 Genomics UK (COG-UK) Consortium. An integrated national scale SARS-CoV-2 genomic surveillance network. Lancet Microbe 1, e99-e100 (2020).

40. Walls, A. C. et al. Structure, function, and antigenicity of the SARS-CoV-2 spike glycoprotein. Cell $\mathbf{1 8 1}$, 281-292.e6 (2020)

41. Wrapp, D. et al. Cryo-EM structure of the 2019-nCoV spike in the prefusion conformation. Science 367 , 1260-1263 (2020). 
42. Lan, J. et al. Structure of the SARS-CoV-2 spike receptor-binding domain bound to the ACE2 receptor Nature 581, 215-220 (2020).

43. Shang, J. et al. Structural basis of receptor recognition by SARS-CoV-2. Nature 581, 221-224 (2020).

44. V'kovski, P., Kratzel, A., Steiner, S., Stalder, H. \& Thiel, V. Coronavirus biology and replication: implications for SARS-CoV-2. Nat. Rev. Microbiol. 19, 155-170 (2020)

45. Carvalho, T., Krammer, F. \& Iwasaki, A. The first 12 months of COVID-19: a timeline of immunological insights. Nat Rev. Immunol 21, 245-256 (2021).

46. Watanabe, Y. et al. Vulnerabilities in coronavirus glycan shields despite extensive glycosylation. Nat. Commun. 11, 2688 (2020).

47. Piccoli, L. et al. Mapping neutralizing and immunodominant sites on the SARS-CoV-2 spike receptor-binding domain by structure-guided highresolution serology. Cell 183, 1024-1042.e21 (2020)

48. Weisblum, Y. et al. Escape from neutralizing antibodies by SARS-CoV-2 spike protein variants. elife 9, e61312 (2020).

49. Wec, A. Z. et al. Broad neutralization of SARS-related viruses by human monoclonal antibodies. Science 369, 731-736 (2020).

50. Greaney, A. J. et al. Comprehensive mapping of mutations in the SARS-CoV-2 receptor-binding domain that affect recognition by polyclonal human plasma antibodies. Cell Host Microbe 29, 463-476.e6 (2021).

This paper demonstrates that a high proportion of neutralizing antibodies elicited by SARS-CoV-2 infection will bind poorly to epitopes containing the spike RBD mutation E484K

51. McCallum, M. et al. N-terminal domain antigenic mapping reveals a site of vulnerability for SARS-CoV-2 Cell 184, 2332-2347.e16 (2021).

52. Harvey, W. T. et al. SARS-CoV-2 variants, spike mutations and immune escape. Nat. Rev. Microbiol. 19, 409-424 (2021).

This paper presents a comprehensive review of SARS-CoV-2 spike immune escape mutations.

53. McMahan, K. et al. Correlates of protection against SARS-CoV-2 in rhesus macaques. Nature 590 , 630-634 (2020)

54. Chandrashekar, A. et al. SARS-CoV-2 infection protects against rechallenge in rhesus macaques. Science 369 812-817 (2020).

55. Baden, L. R. et al. Efficacy and safety of the mRNA-1 273 SARS-CoV-2 vaccine. N. Engl. J. Med. 384, 403-416 (2021).

56. Polack, F. P. et al. Safety and efficacy of the BNT162b2 mRNA COVID-19 vaccine. N. Engl. J. Med. 383, 2603-2615 (2020).

57. Walsh, E. E. et al. Safety and immunogenicity of two RNA-based COVID-19 vaccine candidates. N. Engl. J. Med. 383, 2439-2450 (2020).

58. Sahin, U. et al. COVID-19 vaccine BNT162b1 elicits human antibody and $\mathrm{T}_{\mathrm{H}} 1 \mathrm{~T}$ cell responses. Nature 586, 594-599 (2020).

59. Khoury, D. S. et al. Neutralizing antibody levels are highly predictive of immune protection from symptomatic SARS-CoV-2 infection. Nat. Med. 27 1205-1211 (2021) This paper presents a meta-analysis showing that the level of neutralizing antibodies elicited by a vaccine is strongly correlated with its efficacy.

60. Iwasaki, A. \& Yang, Y. The potential danger of suboptimal antibody responses in COVID-19. Nat. Rev. Immunol. 20, 339-341 (2020).

61. Atyeo, C. et al. Distinct early serological signatures track with SARS-CoV-2 survival. Immunity $\mathbf{5 3}$ 524-532.e4 (2020).

62. Weinreich, D. M. et al. REGN-COV2, a neutralizing antibody cocktail, in outpatients with COVID-19. N. Engl. J. Med. 384, 238-251 (2021).

63. Garcia-Beltran, W. F. et al. COVID-19-neutralizing antibodies predict disease severity and survival. Cell 184, 476-488.e11 (2021)

64. Gottlieb, R. L. et al. Effect of bamlanivimab as monotherapy or in combination with etesevimab on viral load in patients with mild to moderate COVID-19: a randomized clinical trial. JAMA 325, 632-644 (2021)

65. Weinreich, D. et al. REGEN-COV antibody cocktail clinical outcomes study in COVID-19 outpatients. Preprint at medRxiv https://doi.org/ $10.1101 / 2021.05 .19 .21257469$ (2021)

66. Gupta, A. et al. Early COVID-19 treatment with SARS-CoV-2 neutralizing antibody sotrovimab. Preprint at medRxiv https://doi.org/10.1101/ 2021.05.27.21257096 (2021).
67. Corti, D., Purcell, L. A., Snell, G. \& Veesler, D. Tackling COVID-19 with neutralizing monoclona antibodies. Cell 184, 3086-3108 (2021).

68. Rappazzo, C. G. et al. Broad and potent activity against SARS-like viruses by an engineered human monoclonal antibody. Science 371, 823-829 (2021).

69. Pinto, D. et al. Cross-neutralization of SARS-CoV-2 by a human monoclonal SARS-CoV antibody. Nature 583, 290-295 (2020).

70. Cerutti, G et al. Potent SARS-CoV-2 neutralizing antibodies directed against spike $\mathrm{N}$-terminal domain target a single supersite. Cell Host Microbe 29 819-833.e7 (2021).

71. Barnes, C. O. et al. SARS-CoV-2 neutralizing antibody structures inform therapeutic strategies. Nature $\mathbf{5 8 8}$ 682-687 (2020).

This paper describes a structural and functional classification system for neutralizing mAbs targeting the SARS-CoV-2 spike RBD.

72. Finkelstein, M. T. et al. Structural analysis of neutralizing epitopes of the SARS-CoV-2 spike to guide therapy and vaccine design strategies. Viruses 13, 134 (2021)

73. Yuan, M., Liu, H., Wu, N. C. \& Wilson, I. A Recognition of the SARS-CoV-2 receptor binding domain by neutralizing antibodies. Biochem. Biophys. Res. Commun. 538, 192-203 (2021)

74. Dejnirattisai, W. et al. The antigenic anatomy of SARS-CoV-2 receptor binding domain. Cell 184 2183-2200.e22 (2021).

75. Poh, C. M. et al. Two linear epitopes on the SARS-CoV-2 spike protein that elicit neutralising antibodies in COVID-19 patients. Nat. Commun. 11, 2806 (2020)

76. Shiakolas, A. R. et al. Cross-reactive coronavirus antibodies with diverse epitope specificities and Fc effector functions. Cell Rep. Med. 2, 100313 (2021).

77. Wang, C. et al. A conserved immunogenic and vulnerable site on the coronavirus spike protein delineated by cross-reactive monoclonal antibodies. Nat. Commun. 12, 1715 (2021).

78. Schäfer, A. et al. Antibody potency, effector function and combinations in protection and therapy for SARS-CoV-2 infection in vivo. J. Exp. Med. 218 e20201993 (2021).

This study demonstrates the likely importance of SARS-CoV-2 neutralizing mAb Fc-effector functions in an animal model.

79. Winkler, E. S. et al. Human neutralizing antibodies against SARS-CoV-2 require intact Fc effector functions for optimal therapeutic protection. Cell 184 1804-1820.e16 (2021).

80. Tauzin, A. et al. A single dose of the SARS-CoV-2 vaccine BNT162b2 elicits Fc-mediated antibody effector functions and T cell responses. Cell Host Microbe. 29, 1137-1150 (2021)

81. Bartsch, Y. C. et al. Discrete SARS-CoV-2 antibody titers track with functional humoral stability. Nat. Commun. 12, 1018 (2021).

82. Earle, K. A. et al. Evidence for antibody as a protective correlate for COVID-19 vaccine. Vaccine 39 4423-4428 (2021)

83. DiPiazza, A. T., Graham, B. S. \& Ruckwardt, T. J. T cell immunity to SARS-CoV-2 following natural infection and vaccination. Biochem. Biophys. Res. Commun. 538, 211-217 (2020).

84. Sette, A. \& Crotty, S. Adaptive immunity to SARS CoV-2 and COVID-19. Cell 184, 861-880 (2021).

85. Yu, J. et al. DNA vaccine protection against SARS-CoV-2 in rhesus macaques. Science 369 806-811 (2020).

86. Bange, $\mathrm{E}$. M et al. $\mathrm{CD}^{+} \mathrm{T}$ cells contribute to survival in patients with COVID-19 and hematologic cancer. Nat Med. 27, 1280-1289 (2021)

87. Braun, J. et al. SARS-CoV-2-reactive T cells in healthy donors and patients with COVID-19. Nature 587 270-274 (2020)

88. Jackson, L. A. et al. An mRNA vaccine against SARS-CoV-2 - preliminary report. N. Engl. J. Med. 383, 1920-1931 (2020).

89. Grifoni, A et al SARS-CoV-2 Human T cell Epitopes: adaptive immune response against COVID-19. Cell Host Microbe 29, 1076-1092 (2021). This paper presents a systematic review of published SARS-CoV-2 helper and cytotoxic T cell epitopes.

90. Saini, S. K. et al. SARS-CoV-2 genome-wide T cell epitope mapping reveals immunodominance and substantial CD8 ${ }^{+} \mathrm{T}$ cell activation in COVID-19 patients. Sci. Immunol. 6, eabf7550 (2021).
91. Tarke, A. et al. Impact of SARS-CoV-2 variants on the total $\mathrm{CD}^{+}$and $\mathrm{CD} 8+\mathrm{T}$ cell reactivity in infected or vaccinated individuals. Cell Rep. Med. 20, 100355 (2021).

92. Motozono, C. et al. SARS-CoV-2 spike L452R variant evades cellular immunity and increases infectivity. Cell Host Microbe 29, 1124-1136 (2021)

93. Gao, A. et al. Learning from HIV-1 to predict the immunogenicity of T cell epitopes in SARS-COV-2 iScience 24, 102311 (2021).

94. Tarke, A. et al. Comprehensive analysis of T cell immunodominance and immunoprevalence of SARS-CoV-2 epitopes in COVID-19 cases. Cell Rep. Med. 2, 100204 (2021)

95. Dolton, G. et al. Emergence of immune escape at dominant SARS-CoV-2 killer T-cell epitope. Preprint at medRxiv https://doi.org/10.1101/ 2021.06.21.21259010 (2021).

96. Agerer, B. et al. SARS-CoV-2 mutations in MHC-I-restricted epitopes evade $\mathrm{CD} 8^{+} \mathrm{T}$ cell responses. Sci. Immunol. 6, eabg6461 (2021).

97. Chen, $X$ et al Neutralizing antibodies against SARS-CoV-2 variants induced by natural infection or vaccination: a systematic review and pooled meta-analysis. Clin. Infect. Dis. https://doi.org/ 10.1093/cid/ciab646 (2021).

This paper presents a systematic review and meta-analysis of in vitro neutralization studies of SARS-CoV-2 variants by convalescent and vaccinee plasma.

98. Focosi, D., Tuccori, M., Baj, A. \& Maggi, F. SARS-CoV-2 variants: a synopsis of in vitro efficacy data of convalescent plasma, currently marketed vaccines, and monoclonal antibodies. Viruses 13, 1211 (2021).

99. Faulkner, N. et al. Reduced antibody cross-reactivity following infection with B.1.1.7 than with parental SARS-CoV-2 strains. Elife 10, e69317 (2021).

100. Liu, C. et al. Reduced neutralization of SARS-CoV-2 B.1.617 by vaccine and convalescent serum. Cell 184 , 4220-4336 (2021).

101. Moyo-Gwete, T. et al. Cross-reactive neutralizing antibody responses elicited by SARS-CoV-2 501Y.V2 (B. 1.351). N. Engl. J. Med. 384, 2161-2163 (2021). This experimental study shows that the plasma from persons infected with the beta variant can usually neutralize pre-variant viruses.

102. Supasa, P. et al. Reduced neutralization of SARS-CoV-2 B.1.1.7 variant by convalescent and vaccine sera. Cell 184, 2201-2211 (2021).

103. Dejnirattisai, W. et al. Antibody evasion by the P. 1 strain of SARS-CoV-2. Cell 184, 2939-2954 (2021).

104. Zhou, D. et al. Evidence of escape of SARS-CoV-2 variant B. 1.351 from natural and vaccine induced sera. Cell 184, 2348-2361 (2021).

105. Baum, A. et al. Antibody cocktail to SARS-CoV-2 spike protein prevents rapid mutational escape seen with individual antibodies. Science 369, 1014-1018 (2020).

106. Crawford, K. H. D. et al. Protocol and reagents for pseudotyping lentiviral particles with SARS-CoV-2 spike protein for neutralization assays. Viruses 12 , 513 (2020).

107. Schmidt, F. et al. Measuring SARS-CoV- 2 neutralizing antibody activity using pseudotyped and chimeric viruses. J. Exp. Med. 217, e20201181 (2020).

108. Case, J. B. et al. Replication-competent vesicular stomatitis virus vaccine vector protects against SARS-CoV-2-mediated pathogenesis in mice. Cell Host Microbe 28, 465-474.e4 (2020).

109. Copin, R. et al. The monoclonal antibody combination REGEN-COV protects against SARS-CoV- 2 mutational escape in preclinical and human studies. Cell 184 3949-3961 (2021).

110. FDA. Fact sheet for health care providers emergency use authorization (EUA) of bamlanivimab. FDA.gov https://www.fda.gov/media/143603/download (2021).

111. Focosi, D. et al. Emergence of SARS-COV-2 Spike Protein Escape Mutation Q493R after Treatment for COVID-19. Emerg. Infect. Dis. 27 (2021).

112. Choi, B. et al. Persistence and evolution of SARS-CoV-2 in an immunocompromised host. N. Engl. J. Med. 383, 2291-2293 (2020)

113. Kemp, S. A. et al. SARS-CoV-2 evolution during treatment of chronic infection. Nature 592, 277-282 (2021).

114. Avanzato, V. A. et al. Case study: prolonged infectious SARS-CoV-2 shedding from an asymptomatic immunocompromised individual with cancer. Cell 183 1901-1912.e9 (2020)

115. Xie, X. et al. Neutralization of SARS-CoV-2 spike 69/70 deletion, E484K and N501Y variants by BNT $162 \mathrm{~b} 2$ vaccine-elicited sera. Nat. Med. 27, 620-621 (2021). 
116. Liu, Y. et al. BNT162b2-Elicited Neutralization against New SARS-CoV-2 Spike Variants. N. Engl. J. Med. 385, 472-474 (2021).

117. Chen, R. E. et al. Resistance of SARS-CoV-2 variants to neutralization by monoclonal and serum-derived polyclonal antibodies. Nat. Med. 27, 717-726 (2021).

118. Liu, J. et al. BNT162b2-elicited neutralization of B. 1.617 and other SARS-CoV-2 variants. Nature 596, 273-275 (2021).

119. Thi Nhu Thao, T. et al. Rapid reconstruction of SARS-CoV-2 using a synthetic genomics platform. Nature 582, 561-565 (2020)

120. Xie, $X$. et al. Engineering SARS-CoV-2 using a reverse genetic system. Nat. Protoc. 16, 1761-1784 (2021).

121. Wang, P. et al. Antibody resistance of SARS-CoV-2 variants B.1.351 and B.1.1.7. Nature 593, 130-135 (2021).

This paper presents a comprehensive analysis of the effects of Alpha and Beta variant mutations on antibody neutralization.

122. Edara, V. V et al. Infection and Vaccine-Induced Neutralizing-Antibody Responses to the SARS-CoV-2 B. 1.617 Variants. N. Engl. J. Med. 385, 664-666 (2021)

123. Deng, X. et al. Transmission, infectivity, and neutralization of a spike L452R SARS-CoV-2 variant. Cell 184, 3426-3437.e8 (2021)

124. Cele, S. et al. Escape of SARS-CoV-2 501Y.V2 from neutralization by convalescent plasma. Nature 593 142-146 (2021)

125. Riepler, L. et al. Comparison of four SARS-CoV-2 neutralization assays. Vaccines 9, 13 (2021).

126. Sholukh, A. M. et al. Evaluation of cell-based and surrogate SARS-CoV-2 neutralization assays. J. Clin. Microbiol. https://doi.org/10.1128/JCM.00527-21 (2021)

127. Liu, Y. et al. The N501Y spike substitution enhances SARS-CoV-2 transmission. Preprint at bioRxiv https://doi.org/10.1101/2021.03.08.434499 (2021).

128. Starr, T. N. et al. Deep mutational scanning of SARS-CoV-2 receptor binding domain reveals constraints on folding and ACE2 binding. Cell 182, 1295-1310.e20 (2020).

129. Starr, T. N. et al. Prospective mapping of viral mutations that escape antibodies used to treat COVID-19. Science 371, 850-854 (2021).

130. Greaney, A. J. et al. Complete mapping of mutations to the SARS-CoV-2 spike receptor-binding domain that escape antibody recognition. Cell Host Microbe 29, 44-57.e9 (2021).

131. Starr, T. N., Greaney, A. J., Dingens, A. S. \& Bloom, J. D. Complete map of SARS-CoV-2 RBD mutations that escape the monoclonal antibody LY-CoV555 and its cocktail with LY-CoV016. Cell Rep. Med. 2, 100255 (2021)

132. Abe, K. T. et al. A simple protein-based surrogate neutralization assay for SARS-CoV-2. JCI Insight 5 , e142362 (2020).

133. Tan, C. W. et al. A SARS-CoV-2 surrogate virus neutralization test based on antibody-mediated blockage of ACE2-spike protein-protein interaction. Nat. Biotechnol. 38, 1073-1078 (2020).

134. Zhang, J. Z. et al. Detection of antibodies neutralizing historical and emerging SARS-CoV-2 strains using a thermodynamically coupled de novo biosensor system. Preprint at bioRxiv https://doi.org/10.1101/ 2021.06 .22 .449355 (2021)

135. Plante, J. A. et al. Spike mutation D614G alters SARS-CoV-2 fitness. Nature 592, 116-121 (2020).

136. Hou, Y. J. et al. SARS-CoV-2 D614G variant exhibits efficient replication ex vivo and transmission in vivo. Science 370, 1464-1468 (2020).

137. Zhou, B. et al. SARS-CoV-2 spike D614G change enhances replication and transmission. Nature $\mathbf{5 9 2}$ 122-127 (2021)

138. Yurkovetskiy, L. et al. Structural and functional analysis of the D614G SARS-CoV-2 spike protein variant. Cell 183, 739-751.e8 (2020).

139. Benton, D. J. et al. Receptor binding and priming of the spike protein of SARS-CoV-2 for membrane fusion. Nature 588, 327-330 (2020).

140. Gobeil, S. M. C. et al. Effect of natural mutations of SARS-CoV- 2 on spike structure, conformation, and antigenicity. Science 373, eabi6226 (2021).

141. Zhang, L. et al. SARS-CoV-2 spike-protein D614G mutation increases virion spike density and infectivity. Nat. Commun. 11, 6013 (2020).

142. Jackson, C. B., Zhang, L., Farzan, M. \& Choe, H. Functional importance of the D614G mutation in the SARS-CoV-2 spike protein. Biochem. Biophys. Res. Commun. 538, 108-115 (2020).
143. Gobeil, S. M.-C. et al. D614G mutation alters SARS-CoV-2 spike conformation and enhances protease cleavage at the $\mathrm{S} 1 / \mathrm{S} 2$ junction. Cell Rep. 34, 108630 (2021).

144. Weissman, D. et al. D614G spike mutation Increases SARS CoV-2 susceptibility to neutralization. Cell Host Microbe 29, 23-31.e4 (2021).

145. Zou, J. et al. The effect of SARS-CoV-2 D614G mutation on BNT162b2 vaccine-elicited neutralization. NPJ Vaccines 6, 1-4 (2021).

146. Zhu, X. et al. Cryo-electron microscopy structures of the N501Y SARS-CoV-2 spike protein in complex with ACE2 and 2 potent neutralizing antibodies. PLOS Biol. 19, e3001237 (2021).

147. Laffeber, C., de Koning, K., Kanaar, R. \& Lebbink, J. H. G. Experimental evidence for enhanced receptor binding by rapidly spreading SARS-CoV-2 variants. J. Mol. Biol. 433, 167058 (2021).

148. Shen, X. et al. SARS-CoV-2 variant B.1.1.7 is susceptible to neutralizing antibodies elicited by ancestral spike vaccines. Cell Host Microbe 29, 529-539.e3 (2021).

149. Rees-Spear, C. et al. The effect of spike mutations on SARS-CoV-2 neutralization. Cell Rep. 34, 108890 (2021).

150. Edara, V. V. et al. Neutralizing Antibodies Against SARS-CoV-2 Variants After Infection and Vaccination. JAMA 325, 1896-1898 (2021).

151. Wu, K. et al. Serum neutralizing activity elicited by mRNA-1273 vaccine - preliminary report. N. Engl. J. Med. 384, 1468-1470 (2021)

152. Muik, A. et al. Neutralization of SARS-CoV-2 lineage B.1.1.7 pseudovirus by BNT162b2 vaccine-elicited human sera. Science 371, 1152-1153 (2021)

153. Wang, Z. et al. mRNA vaccine-elicited antibodies to SARS-CoV-2 and circulating variants. Nature 592 616-622 (2021)

154. Annavajhala, M. K. et al. A novel SARS-CoV-2 variant of concern, B. 1.526, identified in New York. Preprint at bioRxiv https://doi.org/10.1101/ 2021.02.23.21252259 (2021).

155. Bascos, N. A. D., Mirano-Bascos, D. \& Saloma, C. P. Structural analysis of spike protein mutations in an emergent SARS-CoV-2 variant from the Philippines. Preprint at bioRxiv https://doi.org/10.1101/ 2021.03.06.434059 (2021)

156. Tablizo, F. A. et al. Genome sequencing and analysis of an emergent SARS-CoV-2 variant characterized by multiple spike protein mutations detected from the Central Visayas Region of the Philippines. Preprint at bioRxiv https://doi.org/10.1101/ 2021.03.03.21252812 (2021).

157. Ferrareze, P. A. G. et al. E484K as an innovative phylogenetic event for viral evolution: genomic analysis of the E484K spike mutation in SARS-CoV-2 lineages from Brazil. Infect. Genet. Evol. 93, 104941 (2021).

158. Grabowski, F., Preibisch, G., Giziński, S., Kochańczyk, M. $\&$ Lipniacki, T. SARS-CoV-2 variant of concern 202012/01 has about twofold replicative advantage and acquires concerning mutations. Viruses 13, 392 (2021).

159. Collier, D. A. et al. Sensitivity of SARS-CoV-2 B.1.1.7 to $\mathrm{mRNA}$ vaccine-elicited antibodies. Nature 593 . 136-141 (2021).

160. Liu, Z. et al. Identification of SARS-CoV-2 spike mutations that attenuate monoclonal and serum antibody neutralization. Cell Host Microbe 29, 477-488.e4 (2021).

161. Jangra, S. et al. SARS-CoV-2 spike E484K mutation reduces antibody neutralisation. Lancet Microbe 2 e283-e284 (2021)

162. Tada, T. et al. Convalescent-Phase Sera and Vaccine Elicited Antibodies Largely Maintain Neutralizing Titer against Global SARS-CoV-2 Variant Spikes. mBio 12 e0069621 (2021).

163. Li, Q. et al. The impact of mutations in SARS-CoV-2 spike on viral infectivity and antigenicity. Cell 182 1284-1294.e9 (2020)

164. Greaney, A. J. et al. Antibodies elicited by mRNA1273 vaccination bind more broadly to the recepto binding domain than do those from SARS-CoV-2 infection. Sci. Transl Med. 13, eabi9915 (2021).

165. Ferreira, I. et al. SARS-CoV-2 B. 1.617 mutations L452 and $\mathrm{E} 484 \mathrm{Q}$ are not synergistic for antibody evasion. J. Infect. Dis. https://doi.org/10.1093/infdis/iiab368 (2021).

166. Zhang, L. et al. Comparison of 10 emerging SARS CoV-2 variants: infectivity, animal tropism, and antibody neutralization. Preprint at Research Square https://doi.org/10.21203/rs.3.rs-492659/v1 (2021).

167. Wang, R. et al. Analysis of SARS-CoV-2 variant mutations reveals neutralization escape mechanisms and the ability to use ACE2 receptors from additional species. Immunity 54, 1611-1621.e5 (2021).
168. Chen, J., Wang R.,Wang, M. \& Wei, G.-W. Mutations strengthened SARS-CoV-2 infectivity. J. Mol. Biol. 432, 5212-5226 (2020)

169. Thomson, E. C. et al. Circulating SARS-CoV-2 spike $\mathrm{N} 439 \mathrm{~K}$ variants maintain fitness while evading antibody-mediated immunity. Cell 184, 1171-1187. e20 (2021).

170. Munnink, B. B. O. et al. Transmission of SARS-CoV-2 on mink farms between humans and mink and back to humans. Science 371, 172-177 (2021).

171. Bayarri-Olmos, R. et al. The SARS-CoV-2 Y453F mink variant displays a pronounced increase in ACE- 2 affinity but does not challenge antibody neutralization J. Biol. Chem. 296, 100536 (2021).

172. Hodcroft, E. B. et al. Spread of a SARS-CoV-2 variant through Europe in the summer of 2020. Nature 595 707-712 (2021)

173. Giacomo, S. D., Mercatelli, D., Rakhimov, A. \& Giorgi, F. M. Preliminary report on severe acute respiratory syndrome coronavirus 2 (SARS-CoV-2) Spike mutation T478K. J. Med. Virol. 93, 5638-5643 (2021).

174. Muecksch, F. et al. Affinity maturation of SARS-CoV-2 neutralizing antibodies confers potency, breadth, and resilience to viral escape mutations. Immunity $\mathbf{5 4}$ 1853-1868.e7 (2021).

175. Cathcart, A. L. et al. The dual function monoclonal antibodies VIR-7831 and VIR-7832 demonstrate potent in vitro and in vivo activity against SARS-CoV-2 Preprint at bioRxiv https://doi.org/10.1101/ 2021.03.09.434607 (2021).

176. McCarthy, K. R. et al. Recurrent deletions in the SARS-CoV-2 spike glycoprotein drive antibody escape. Science 371, 1139-1142 (2021)

177. Meng, B. et al. Recurrent emergence of SARS-CoV-2 spike deletion $\mathrm{H} 69 / \mathrm{V} 70$ and its role in the alph variant B.1.1.7. Cell Rep. 35, 109292 (2021).

178. Chi, X. et al. A neutralizing human antibody binds to the $\mathrm{N}$-terminal domain of the spike protein of SARS-CoV-2. Science 369, 650-655 (2020).

179. Liu, L. et al. Potent neutralizing antibodies against multiple epitopes on SARS-CoV-2 spike. Nature 584 450-456 (2020).

180. Hodcroft, E. B. et al. Emergence in late 2020 of multiple lineages of SARS-CoV-2 spike protein variants affecting amino acid position 677. Preprint at bioRxiv https:// doi.org/10.1101/2021.02.12.21251658 (2021).

181. Brown, J. C. et al. Increased transmission of SARS-CoV-2 lineage B.1.1.7 (VOC 2020212/01) is not accounted for by a replicative advantage in primary airway cells or antibody escape. Preprint at bioRxiv https://doi.org/10.1101/2021.02.24.432576 (2021).

182. Johnson, B. A. et al. Loss of furin cleavage site attenuates SARS-CoV-2 pathogenesis. Nature 591 , 293-299 (2021)

183. Saito, A. et al. SARS-CoV-2 spike P681R mutation enhances and accelerates viral fusion. Preprint at bioRxiv https://doi.org/10.1101/2021.06.17.448820 (2021).

184. Guo, K. et al. Interferon resistance of emerging SARS-CoV-2 variants. Preprint at bioRxiv https:/ doi.org/10.1101/2021.03.20.436257 (2021)

185. Thorne, L. G. et al. Evolution of enhanced innate immune evasion by the SARS-CoV-2 B.1.1.7 UK variant. Preprint at bioRxiv https://doi.org/10.1101/ 2021.06.06.446826 (2021).

This systems biology study shows that in contrast to earlier SARS-CoV-2 variants, the alpha variant may owe its increased transmissibility and virulence to its antagonism of cellular innate immunity.

186. Jiang, H. et al. SARS-CoV-2 Orf9b suppresses type interferon responses by targeting TOM70. Cell Mol. Immunol. 17, 998-1000 (2020).

187. Parker, M. D. et al. Altered sub-genomic RNA expression in SARS-CoV-2 B.1.1.7 infections. Preprint at bioRxiv https://doi.org/10.1101/ 2021.03.02.433156 (2021).

188. Xia, H. et al. Evasion of type I interferon by SARS-CoV-2. Cell Rep. 33, 108234 (2020).

189. Plante, J. A. et al. The variant gambit: COVID-19's next move. Cell Host Microbe 29, 508-515 (2021).

190. Leary, S. et al. Generation of a novel SARS-CoV-2 sub-genomic RNA due to the R203K/G204R variant in nucleocapsid. Preprint at bioRxiv https://doi.org/ 10.1101/2020.04.10.029454 (2021).

191. Volz, E. et al. Assessing transmissibility of SARS-CoV-2 lineage B.1.1.7 in England. Nature 593, 266-269 (2021).

This analysis demonstrates the increased transmissibility of the alpha variant

192. Peng, J. et al. Estimation of secondary household attack rates for emergent spike L452R SARS-CoV-2 variants 
detected by genomic surveillance at a community-based testing site in San Francisco. Clin. Infect. Dis. https:/ doi.org/10.1093/cid/ciab283 (2021).

193. Allen, H. et al. Increased household transmission of COVID-19 cases associated with SARS-CoV-2 variant of concern B.1.617.2: a national case-control study. Knowledge Hub https://khub.net/documents/135939561/ 405676950/Increased+Household+Transmissionof+COVID-19+Cases+-+national+case+study.pdf/ 7f7764fb-ecb0-da31-77b3-b1 a8ef7be9aa (2021).

194. Davies, N. G. et al. Increased mortality in communitytested cases of SARS-CoV-2 lineage B.1.1.7. Nature 593, 270-274 (2021).

195. Challen, R. et al. Risk of mortality in patients infected with SARS-CoV-2 variant of concern 202012/1: matched cohort study. BMJ 372, n579 (2021).

196. Mullen, J. L. et al. Outbreak.info https://outbreak.info/ citation (2020)

197. Leung, K., Shum, M. H., Leung, G. M., Lam, T. T. \& Wu, J. T. Early transmissibility assessment of the N501Y mutant strains of SARS-CoV-2 in the United Kingdom, October to November 2020. Eur. Surveill. 26, 2002106 (2021)

198. Davies, N. G. et al. Estimated transmissibility and impact of SARS-CoV-2 lineage B.1.1.7 in England Science 372, eabg3055 (2021).

199. Golubchik, T. et al. Early analysis of a potential link between viral load and the N501Y mutation in the SARS-COV-2 spike protein. Preprint at bioRxiv https:// doi.org/10.1101/2021.01.12.20249080 (2021).

200. Kidd, M. et al. S-variant SARS-CoV-2 lineage B1.1.7 is associated with significantly higher viral loads in samples tested by ThermoFisher TaqPath RT-qPCR. J. Infect. Dis. 223, 1666-1670 (2021).

201. Borges, V. et al. Tracking SARS-CoV-2 lineage B. 1.1.7 dissemination: insights from nationwide spike gene target failure (SGTF) and spike gene late detection (SGTL) data, Portugal, week 492020 to week 32021. Eur. Surveill. 26, 2100131 (2021)

202. Grint, D. J. et al. Case fatality risk of the SARS-CoV-2 variant of concern B.1.1.7 in England, 16 November to 5 February. Eur. Surveill. 26, 2100256 (2021).

203. Betton, M. et al. Sera neutralizing activities against SARS-CoV-2 and multiple variants six month after hospitalization for COVID-19. Clin. Infect. Dis. https://doi.org/10.1093/cid/ciab308 (2021).

204. Planas, D et al. Sensitivity of infectious SARS-CoV-2 B. 1.1.7 and B. 1.351 variants to neutralizing antibodies. Nat. Med. 27, 917-924 (2021).

205. Edara, V. V., Hudson, W. H., Xie, X., Ahmed, R. $\&$ Suthar, M. S. Neutralizing antibodies against SARS-CoV-2 variants after infection and vaccination. JAMA 325, 1896-1898 (2021).

206. Wang, G.-L. et al. Susceptibility of circulating SARS-CoV-2 variants to neutralization. N. Engl. J. Med. 384, 2354-2356 (2021)

207. Graham, M. S. et al. Changes in symptomatology, reinfection, and transmissibility associated with the SARS-CoV-2 variant B. 1.1.7: an ecological study. Lancet Public Health 6, e335-e345 (2021).

208. Garcia-Beltran, W. F. et al. Multiple SARS-CoV-2 variants escape neutralization by vaccine-induced humoral immunity. Cell 184, 2372-2383.e9 (2021).

209. Wall, E. C. et al. Neutralising antibody activity against SARS-CoV-2 VOCs B.1.617.2 and B. 1.351 by BNT162b2 vaccination. Lancet 397, 2331-2333 (2021).

210. Haas, E. J. et al. Impact and effectiveness of mRNA BNT 162 b2 vaccine against SARS-CoV-2 infections and COVID-19 cases, hospitalisations, and deaths following a nationwide vaccination campaign in Israel: an observational study using national surveillance data. Lancet 397, 1819-1829 (2021).

211. Abu-Raddad, L. J., Chemaitelly, H. \& Butt, A. A Effectiveness of the BNT162b2 Covid-19 Vaccine against the B.1.1.7 and B.1.351 Variants. N. Engl. J. Med. 385, 187-189 (2021).

212. Heath, P. T. et al. Safety and efficacy of NVX-CoV2373 Covid-19 vaccine. N. Engl. J. Med. https://doi.org/ 10.1056/NEJMoa2107659 (2021)

213. Emary, K. R. W. et al. Efficacy of ChAdOx 1 nCoV-19 (AZD 1222) vaccine against SARS-CoV-2 variant of concern 202012/01 (B.1.1.7): an exploratory analysis of a randomised controlled trial. Lancet 397, 1351-1362 (2021).

214. Planas, D. et al. Reduced sensitivity of SARS-CoV-2 variant Delta to antibody neutralization. Nature 596, 276-280 (2021).

215. Mlcochova, P. et al. SARS-CoV-2 B.1.617.2 Delta variant emergence and vaccine breakthrough. Preprin at Research Square https://www.researchsquare.com/ article/rs-637724/v1 (2021).
216. Shinde, V. et al. Efficacy of NVX-CoV2373 Covid-19 vaccine against the B.1.351 variant. N. Engl. J. Med. 384, 1899-1909 (2021).

This post-hoc analysis of a clinical trial shows that the beta variant was frequently responsible for reinfections in South Africa.

217. Hoffmann, M. et al. SARS-CoV-2 variants B. 1.35 and P. 1 escape from neutralizing antibodies. Cell 184 2384-2393.e12 (2021).

218. Wibmer, C. K. et al. SARS-CoV-2 501Y.V2 escapes neutralization by South African COVID-19 donor plasma. Nat. Med. 27, 622-625 (2021).

219. Edara, V. V. et al. Infection- and vaccine-induced antibody binding and neutralization of the B. 1.351 SARS-CoV-2 variant. Cell Host Microbe 29, 516-521.e3 (2021).

220. Li, Q. et al. SARS-CoV-2 501Y.V2 variants lack higher infectivity but do have immune escape. Cell $\mathbf{1 8 4}$, 2362-2371.e9 (2021)

221. McCallum, M. et al. SARS-CoV-2 immune evasion by the B.1.427/B.1.429 variant of concern. Science 373 648-654 (2021)

222. Shen, X. et al. Neutralization of SARS-CoV-2 variants B.1.429 and B.1.351. N. Engl. J. Med. 384 , 2352-2354 (2021).

223. Goel, R. R. et al. Distinct antibody and memory B cell responses in SARS-CoV-2 naïve and recovered individuals following mRNA vaccination. Sci. Immunol. 6, eabi6950 (2021)

224. Madhi, S. A. et al. Efficacy of the ChAdOx1 nCoV-19 Covid-19 vaccine against the B. 1.351 variant. N. Engl. J. Med. 384, 1885-1898 (2021). This post-hoc analysis of a clinical trial shows that AZD1 222 appeared ineffective at preventing infection with the beta variant.

225. Sadoff, J. et al. Safety and efficacy of single-dose Ad26.COV2.S vaccine against COVID-19. N. Engl. J. Med. 384, 2187-2201 (2021)

226. Kustin, T. et al. Evidence for increased breakthrough rates of SARS-CoV-2 variants of concern in BNT162b2-mRNA-vaccinated individuals. Nat. Med. 27, 1379-1384 (2021).

227. FDA. Fact sheet for health care providers emergency use authorization (EUA) of bamlanivimab and etesevimab. FDA.gov https://www.fda.gov/media/ 145802/download (2021)

228. Wang, P. et al. Increased resistance of SARS-CoV-2 variant P. 1 to antibody neutralization. Cell Host Microbe 29, 747-751.e4 (2021).

229. Souza, W. M. et al. Neutralisation of SARS-CoV-2 lineage P. 1 by antibodies elicited through natural SARS-CoV-2 infection or vaccination with an inactivated SARS-CoV-2 vaccine: an immunological study. Lancet Microbe. https://doi.org/10.1016/ S2666-5247(21)00129-4 (2021)

230. Pegu, A. et al. Durability of mRNA-1 273 vaccine-induced antibodies against SARS-CoV-2 variants. Science https:// doi.org/10.1126/science.abj4176 (2021).

231. Yadav, P. D. et al. Neutralization of Beta and Delta variant with sera of COVID- 19 recovered cases and vaccinees of inactivated COVID-19 vaccine BBV152/ Covaxin2. J. Travel. Med. https://doi.org/10.1093/jtm/ taab 104 (2021).

232. Bernal, J. L. et al. Effectiveness of COVID-19 vaccines against the B.1.617.2 variant. N. Engl. J. Med. 385 585-594 (2021)

233. Sheikh, A., McMenamin, J., Taylor, B. \& Robertson, C. SARS-CoV-2 Delta VOC in Scotland: demographics, risk of hospital admission, and vaccine effectiveness. Lancet 397, 2461-2462 (2021)

234. Hoffmann, M. et al. SARS-CoV-2 variant B.1.617 is resistant to bamlanivimab and evades antibodies induced by infection and vaccination. Cell Rep. 36 109415 (2021).

235. Zhou, H. et al. B. 1.526 SARS-CoV-2 variants identified in new york city are neutralized by vaccine-elicited and therapeutic monoclonal antibodies. mBio https:// doi.org/10.1128/mBio.01386-21 (2021).

236. Ozer, E. A. et al. High prevalence of SARS-CoV-2 B.1.1.7 (UK variant) and the novel B.1.525 lineage in Oyo State, Nigeria. Preprint at medRxiv https:// doi.org/10.1101/2021.04.09.21255206 (2021).

237. Wink, P. L. et al. First identification of SARS-CoV-2 Lambda (C.37) variant in southern Brazil. Preprint at medRxiv https://doi.org/10.1101/2021.06.21.21259241 (2021).

238. Acevedo, M. L. et al. Infectivity and immune escape of the new SARS-CoV-2 variant of interest Lambda. Preprint at medRxiv https://doi.org/10.1101/ 2021.06.28.21259673 (2021).

239. Wang, Z. et al. Naturally enhanced neutralizing breadth against SARS-CoV-2 one year after infection. Nature 595, 426-431 (2021).
240. Turner, J. S. et al. SARS-CoV-2 mRNA vaccines induce persistent human germinal centre responses. Nature https://doi.org/10.1038/s41586-021-03738-2 (2021).

241. Collier, D. A. et al. Age-related immune response heterogeneity to SARS-CoV-2 vaccine BNT162b2 Nature https://doi.org/10.1038/s41586-021-03739-1 (2021).

242. Furer, V. et al. Immunogenicity and safety of the BNT162b2 mRNA COVID-19 vaccine in adult patients with autoimmune inflammatory rheumatic diseases and in the general population: a multicentre study. Ann. Rheum. Dis. https://doi.org/10.1136/ annrheumdis-2021-220647 (2021).

243. Haidar, G. et al. Immunogenicity of COVID-19 vaccination in immunocompromised patients: an observational, prospective cohort study interim analysis. Preprint at medRxiv https://doi.org/10.1101/ 2021.06.28.21259576 (2021).

244. Nadesalingam, A. et al. Paucity and discordance of neutralising antibody responses to SARS-CoV-2 VOCs in vaccinated immunodeficient patients and health-care workers in the UK. Lancet Microbe https:/ doi.org/10.1016/S2666-5247(21)00157-9 (2021).

245. Krause, P. R. et al. SARS-CoV-2 variants and vaccines. N. Engl. J. Med. 385, 179-186 (2021).

246. Wu, K. et al. Variant SARS-CoV-2 mRNA vaccines confer broad neutralization as primary or booster series in mice. Preprint at bioRxiv https://doi.org/ 10.1101/2021.04.13.439482 (2021).

247. Röltgen, K. \& Boyd, S. D. Antibody and B cell responses to SARS-CoV-2 infection and vaccination. Cell Host Microbe 29, 1063-1075 (2021).

248. Voysey, M. et al. Safety and efficacy of the ChAdOx $\mathrm{nCoV}-19$ vaccine (AZD 1222) against SARS-CoV-2 an interim analysis of four randomised controlled trials in Brazil, South Africa, and the UK. Lancet 397, 99-111 (2020).

249. Ranzani, O. T. et al. Effectiveness of the CoronaVac vaccine in the elderly population during a $P .1$ variantassociated epidemic of COVID-19 in Brazil: a testnegative case-control study. Preprint at medRxiv https://doi.org/10.1101/2021.05.19.21257472 (2021).

\section{Acknowledgements}

The authors thank B. A. Pinsky (Stanford University), S. D. Boyd (Stanford University) and J. D. Bloom (University of Washington, Seattle, WA) for critical review of a manuscript draft.

\section{Author contributions}

K.T., P.L.T. and R.W.S. conceived of the project. K.T., P.L.T., J.N. and R.W.S. reviewed the primary literature. K.T. and P.L.T. created the database software necessary for the project. K.T. reviewed the primary data presented in the Review. K.T., P.L.T. and R.W.S. analysed the primary data presented in the manuscript. R.W.S. wrote the manuscript. D.F assisted with creating manuscript figures. R.K.G., T.d.O., S.L.K.-P. and D.F. reviewed multiple drafts of the manuscript.

\section{Competing interests}

R.W.S. has received grant funding from Janssen Pharmaceuticals, Vela Diagnostics and Insilixa; and honoraria from Gilead Sciences and GlaxoSmithKline (CSK). R.K.G. has received honoraria for consulting with Janssen, ViiV and CSK. The other authors declare no competing interests.

\section{Peer review information}

Nature Reviews Genetics thanks J. Dufloo, O. Schwartz and the other, anonymous, reviewer(s) for their contribution to the peer review of this work.

\section{Publisher's note}

Springer Nature remains neutral with regard to jurisdictional claims in published maps and institutional affiliations.

\section{Supplementary information}

The online version contains supplementary material available at https://doi.org/10.1038/s41576-021-00408-x.

\section{RELATED LINKS}

GISAID (Global Initiative on Sharing Avian Influenza Data) Tracking of SARS-CoV-2 Variants: https://www.gisaid.org/ hcov19-variants/ NextStrain - Genomic epidemiology of novel coronavirus: Outbreak.info: https://outbreak.info/

Stanford University Coronavirus Antiviral \& Resistance Database - Susceptibility Data: https://covdb.stanford.edu/ page/susceptibility-data/ WHO - Tracking SARS-CoV-2 Variants: https:// int/en/activities/tracking-SARS-CoV-2-variants/

(c) Springer Nature Limited 2021 